\title{
Nanotoxicity: Exploring the Interactions Between Carbon Nanotubes and Proteins
}

\author{
Guanghong Zuo ${ }^{1,2}$, Haiping Fang 1,2 and Ruhong Zhou ${ }^{3,4}$ \\ ${ }^{1}$ Shanghai Institute of Applied Physics, Chinese Academy of Sciences, Shanghai, \\ ${ }^{2}$ T-Life Research Center, Department of Physics, Fudan University, Shanghai, \\ ${ }^{3}$ IBM Thomas J. Watson Research Center, Yorktown Heights, NY, \\ ${ }^{4}$ Department of Chemistry, Columbia University, New York, \\ 1,2China \\ 3,4 USA
}

\section{Introduction}

Carbon nanotubes (CNTs), which are entirely composed of $s p^{2}$ carbons, can be viewed as graphite sheets that have been rolled into seamless cylinders. Since their discovery in 1991 [1], CNTs have become the "superstar" in the field of nanoscience and nanotechnology because of their unique structural, mechanical, and electronic properties [2]. Various promising biomedical applications have also been proposed, such as drug design [3], drug delivery [4], tumor therapy [5], tissue engineering [6], DNA recognition [7], and biosensor design [8]. It was predicted that the global market for CNTs could grow to $\$ 2$ billion by 2014 [9].

On the other hand, with the fast advance of the nanotechnology and its widespread applications in various industries, there is a growing concern on the biosafety of these nanomaterials to human health [10-17]. Human skin, lungs, and gastrointestinal tract are in constant contact with the environment. Despite the skin is an effective barrier to foreign substances, the lungs and gastrointestinal tract are vulnerable. Recent experiments showed that unrefined CNTs might be aerosolized and release fine particles into air [18]. When the concentration of CNTs in air is sufficiently high, they can be inhaled and then migrate into other parts of body [19]. These inhaled CNTs can enter cells and accumulate in cytoplasms [20, 21], which may lead to the lung insult [22-24], immunologic toxicity [25], and adverse cardiovascular effects (on mice) [26]. Injections and implants, which are required for some biomedical applications, are the other possible routes of the exposure of human cells to nanoparticles. The experiments on mice showed that the tissue exposing directly to the injected CNTs may lead to asbestos-like pathogenic behaviors, including inflammation and the formation of lesions known as granulomas [27]. In addition, the injecting CNTs into the tail vein of male mice may cause the reversible testis damage [28]. Therefore, there is an urgent need for the understanding of nanotoxicity and the development of biocompatible nanomaterials.

Recently, the interactions between nanoparticles, such as CNTs, and biological molecules, such as proteins, have received great attention due to the aforementioned biosafety concerns. Proteins are the functional units of life. Studies on the interaction of CNTs and 
proteins may provide a key to understand the basic questions in the nanotoxicology and nanopharmacology. In 1999, Mioskowski and coworkers observed that the protein streptavidin can grow into helical crystals on the surface of CNTs in experiment [29]. In 2003, Park et al. showed experimentally that the CNT is effective in blocking some biological membrane ion channels due to its structural match in size and shape [30]. Using atomic force microscope (AFM) and Fourier transform infrared spectroscopy (FTIR), Karajanagi et al. investigated the conformational changes of two enzymes, a-chymotrypsin (CT) and soybean peroxidase (SBP), after adsorbing onto the SWCNT [31]. They argued that the distribution of the hydrophobic residues on the surface of the enzymes may determine the protein conformational changes when adsorbed onto the CNT. The important role of hydrophobic residues during the interactions between proteins and CNTs was also found by the experiments of Goldberg-Oppenheimer and Regev [32], in which they explored the conformational changes of bovine serum albumin (BSA) bound with CNTs via Cryogenic temperature transmission electron microscopy (Cryo-TEM). And the experiments on the interactions between small peptides and CNTs also showed that the tryptophan residues play a key role in the binding of the peptides on CNTs $[33,34]$.

In addition to these studies mentioned above, various experimental techniques have also been developed to shed light on this challenging problem [35-37]. However, the mechanism, particularly the effect of CNTs on the structure and function of biomolecules which may lead to the loss of the original function of proteins, is far from being understood. The inherent difficulties involved in these complex settings can limit their applications due to too many factors involved ("side effects") and experimental results may vary for the same proteins interacting with the same nanoparticles (such as same chemical composition, same shape, length, and aggregation property, etc.) under same conditions (such as same concentration, temperature, and pressure, etc). The computational simulations, on the other hand, might mimic and model this kind of interactions and avoid some complicated side effects. Molecular dynamics (MD) is one of these computational techniques, which is widely used in the studies of biomolecules [38-55], and nanoscale systems [56-60]. It is found effective in providing insights to the interactions between proteins and CNTs. For example, a recent MD simulation revealed some stepwise conformational changes of the sub-domain of human serum albumin (HSA) due to its adsorption onto the CNT surfaces [61].

In this chapter, we will review some of our recent works on the interactions between singlewall carbon nanotubes (SWCNTs) and proteins, and their effects on protein structure and function, using large scale molecular dynamics simulations. It is widely accepted that specific interactions between proteins and other molecules, often based on the structures of active sites, play a vital role in the function of proteins. Thus, if a SWCNT can occupy or disrupt the active site of a protein, it will very likely affect the function of the protein. First, we investigated the probability of the SWCNT disrupting the active site by using a signaling and regulatory protein WW domain (a relatively small protein) as an example. It was found that the SWCNT could plug into the hydrophobic cores of WW domains. Two key residues forming the functional scaffold in the native structure were separated by the SWCNT. This plugging of the SWCNT seriously broke the structure of the active site and reduced the possibility of the WW domain in binding with its partners. Second, we investigated the three-way binding competition among a SWCNT, protein SH3 domain (another small signaling and regulatory protein domain), and a proline rich motif ligand. It was found that the SWCNT had a very high probability occupying the active site of the $\mathrm{SH} 3$ domain, which 
prevented the ligand from binding to the active site. In these two cases, the effects of the SWCNT on the proteins would lead to the loss of the original function of the proteins. Finally, the interactions between the SWCNT and human blood serum proteins, including BSA (bovine serum albumin), $\gamma$-Ig (gamma-globulin), Tf (transferring), and BFg (fibrinogen), were also investigated by MD simulations, to confirm that the mechanisms identified with these small protein domains, such as WW domains and SH3 domains, also apply to much larger proteins. It is found that the hydrophobic interactions between the SWCNTs and nonpolar residues, particularly those aromatic ones through so-called $\pi-\pi$ stacking, play a key role in the interaction between the SWCNTs and proteins.

\section{Results}

\subsection{Disruption of protein active sites}

Proteins are molecular machines, building blocks, and arms of living cells. The enormous variety of protein functions is based on their high specificity in partnering with other molecules, with which they interact -- a relationship that resembles a key and lock. This specific relationship demands a fairly unique spatial structure of the protein, in which the hydrophobic residues are buried in the core (named hydrophobic core). The pristine CNTs are extremely hydrophobic. Thus it is possible for the pristine CNTs to plug into the hydrophobic core due to the strong affinity between the CNT and the hydrophobic residues in the core. This plugging will lead to the loss of the original function of the protein, and thus is a potential mechanism for the toxicity of the CNTs (and maybe other hydrophobic nanoparticles).

We take a WW domain interacting with various sized SWCNTs as an example to illustrate this idea [62]. The WW domain is a protein module in signaling and regulatory proteins as the functional module to identify and bind the proline-rich motifs (PRMs) of its binding partner [63-67]. They exist as a triple stranded anti-parallel $\beta$-sheet structure [68-70]. Two highly conserved aromatic residues, a tryptophan residue in the third $\beta$-strand and an aromatic residue in the second $\beta$-strand, are together in the native structure to form the scaffold to bind the proline residue in the PRM. For the WW domain used in our simulation, which is a module of the human Yes-associated protein [71], the two key residues are W39 at the third $\beta$-strand and Y28 in the second $\beta$-strand. The SWCNTs used in the simulation are armchair of $(m, n)$, where $m=n=(4,5,6)$, corresponding to the tube diameters of $(5.38$ $\AA, 6.73 \AA, 8.08 \AA$ ), respectively. The SWCNT and protein are initially well separated, with a distance between the geometric centre of protein domain and the SWCNT of $15.0 \AA$. Each system was solvated with TIP3P model water. We performed 36 independent simulations for the YAP65 WW domain and these three sizes of SWCNTs with different initial velocities, each with 200 ns (see more detail in Computational Methods).

\section{Interaction of YAP65 WW domain with SWCNT}

In our simulations, we found several cases that the SWCNTs plugged into the YAP65 WW domain between the second and third $\beta$-strands, as one representative structure shown in Fig. 1(a). Fig. 1(b) shows the superposition of the structure of the WW domain in the complex and in the native state. It was found that the main change of the protein conformation in the complex was its third $\beta$-strand. It unfolds into a loop and wraps on the SWCNT. Most of the contacts between the second and third $\beta$-strand are broken. Essentially, these conformational changes of the WW domain are pre-requirements for the 
formation of this complex of the protein domain and the SWCNT. In the complex, nine residues in the second and third $\beta$-strands contact with the SWCNT. Most of them are hydrophobic residues, such as W39. This implies that the hydrophobic interaction, the dominant force for protein folding [72], is the key to this phenomenon, in which the SWCNT plugged into the hydrophobic core of the WW protein domain to form a stable protein-SWCNT complex.
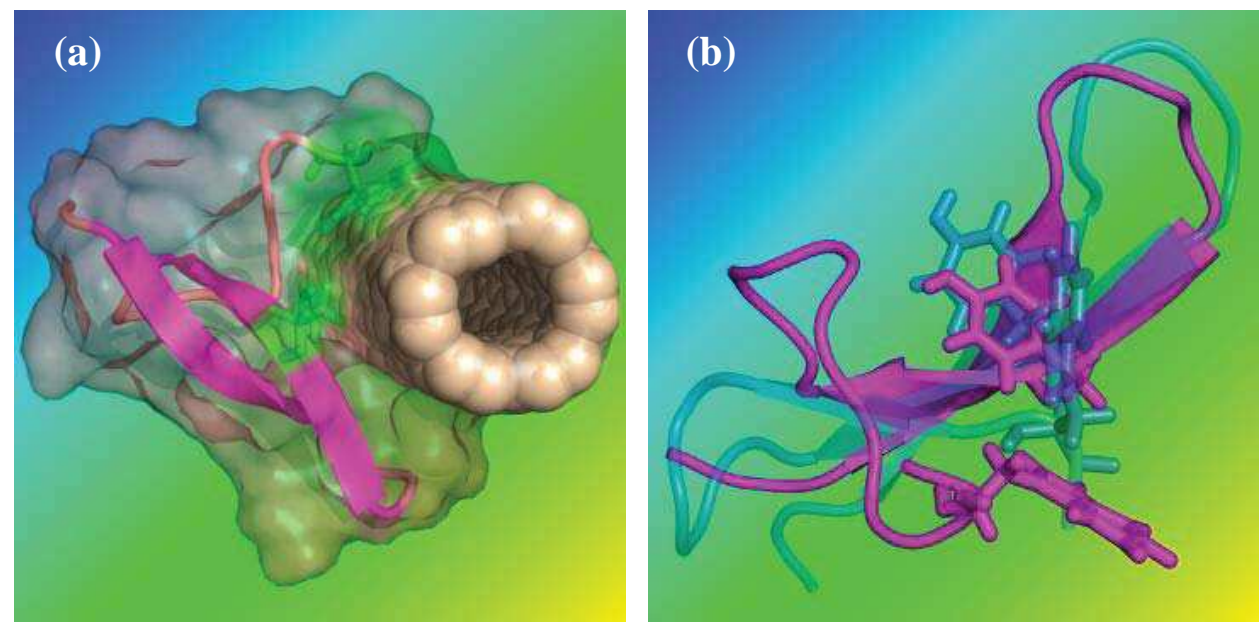

Fig. 1. Typical structures of (a) a complex of the SWCNT plugging into the YAP65 WW domain and (b) The WW domain in the complex (magenta and solid) superposed with its native state (cyan and half semitransparent). In the complex, the YAP65 WW domain is shown in cartoon with magenta strand and salmon loop, and the key residues (W39 and Y28) are identified by green sticks. The $(6,6)$ armchair SWCNT has a diameter of $8.08 \AA$, and its atoms are shown in wheat spheres. The solvated surfaces of the complex are shown in semitransparent.

Fig. 2(a) displays some representative snapshots of these complexes at different times to show the plugging process of the SWCNT. The interface area between the protein domain and the SWCNT (denoted by S, shown in Fig. 2(b)) is used to illustrate this process. Here $S$ is defined as half of the difference between the solvent accessible surface area of the complex and the sum of solvent accessible surface areas of the protein and the SWCNT [73]. Thus at $\mathrm{t}=0, \mathrm{~S}$ is small $\left(\sim 50 \AA^{2}\right)$ since the YAP65 WW domain and the SWCNT are initially separated. The YAP65 WW domain and the SWCNT approached each other very quickly. As a result, $\mathrm{S}$ rose to about $200 \AA^{2}$ within only $1 \mathrm{~ns}$. This indicated that the SWCNT was adsorbed onto the protein surface (see the snapshot of $t=1$ ns in Fig. 2(a)). In the next 45 ns, S gradually increased from $\sim 200 \AA^{2}$ to $\sim 250 \AA^{2}$, with significant fluctuations along the way. It was found that the contacting surface region of the domain kept changing during the process of adsorption. That is, the SWCNT was constantly seeking for a more stable binding site. Around $t=45 \mathrm{~ns}$, there is a negative impulse in the interface area $\mathrm{S}$. A careful examination on the trajectory showed that the SWCNT began to plug into the second and third $\beta$-strands and the negative impulse was a result of the opening of the second and the third $\beta$-strands before the SWCNT was "swallowed" by the protein. S reached its maximal 
value of $\sim 300 \AA^{2}$ at around $\mathrm{t}=80 \mathrm{~ns}$, meaning that the SWCNT was finally successful in plugging into the protein domain. In these structures, the SWCNT was basically wrapped by the second and the third $\beta$-strands, forming a complex of the WW domain with the SWCNT. From $t=80$ ns up to $200 \mathrm{~ns}$, there were only minor fluctuations of $S$ at $\sim 300 \AA^{2}$, showing the stability of this protein-SWCNT complex (data after 100 ns were not shown since the plugging was mainly done in the first $100 \mathrm{~ns}$ ).
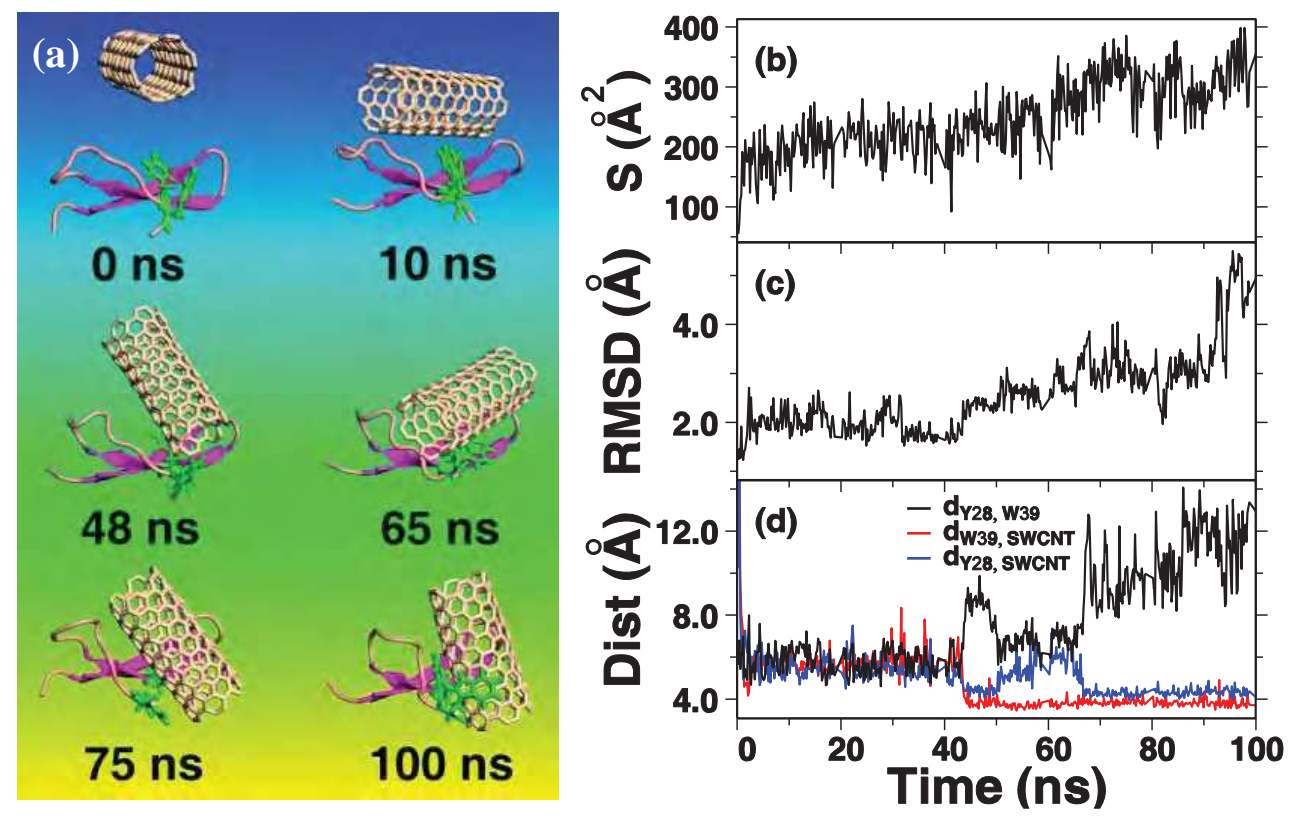

Fig. 2. A typical trajectory of the YAP65 WW domain together with a SWCNT.

(a) Representative snapshots at various time points. The proteins are shown in cartoon with magenta strand and salmon loop. The binding scaffold residues are shown in green sticks and the SWCNT are shown in wheat sticks. (b) The interface area of the WW domain and the SWCNT, (c) The RMSD of the YAP65 WW domain from its native structure, and (d) The distances between the SWCNT and the key residues of YAP65 WW domain, W39 (red) and Y28 (blue), and distance between these two residues (black), denoted by $\mathrm{d}_{\mathrm{W} 39}$, SWCNT, $\mathrm{d}_{\mathrm{Y} 28,}$, SWCNT, and $\mathrm{d}_{\mathrm{W} 39}$, Y28, respectively, as a function of time.

In order to investigate the conformational change of the YAP65 WW domain, we computed the RMSD from its native structure in the MD trajectory, as shown in Fig. 2(c). As a comparison, we had also performed simulations for the YAP65 WW domain only (without any SWCNT or ligand). It was found that without the disruption of the SWCNT, the RMSD of the WW domain remained at a value of around $2.0 \AA$. From the RMSD shown in Fig. 2(c), the conformational change of the YAP65 WW domain can be described as four stages. In the first stage, from 0 to $\sim 45 \mathrm{~ns}$, the RMSD maintained at roughly $2.0 \AA$. That is, there was little impact of the SWCNT on the conformation of the YAP65 WW domain despite its adsorption on the SWCNT surface. In the second stage, from $\sim 45$ to $\sim 70 \mathrm{~ns}$, the RMSD increased gradually from $\sim 2.0 \AA$ to $\sim 3.5 \AA$, which was a notable 
conformational change and corresponded to the opening of the second and third $\beta$-strands due to the insertion of the SWCNT. The third stage is from $\sim 70$ to $\sim 90 \mathrm{~ns}$. In this stage, a small plateau for the RMSD, around $3.0 \AA$, appeared. It was due to the partial recovery of the contacts between the second and third $\beta$-strands. Around $90 \mathrm{~ns}$, there was a significant jump in the RMSD, from $\sim 3.0$ to $\sim 4.0 \AA$, which initiates the fourth stage. Detailed studies showed that there was a change in both the orientation and binding site of the indole ring of W39 residue on the SWCNT during this jumping process. From $\mathrm{t}=\sim 95 \mathrm{~ns}$, the RMSD kept roughly constant at $\sim 4.0 \AA$, indicating the final complex of the YAP65 WW domain and SWCNT was quite stable. This relatively large RMSD indicates a significant conformational alteration of the YAP65 WW domain caused by the insertion of the SWCNT.

The W39 and Y28 are the key residues for the function of the YAP65 WW domain. The interaction between the SWCNT and these two key residues were also investigated. Fig. 2(d) shows the distances between these two residues, and distances between each residue with the SWCNT as a function of time, denoted by $d_{W 39, Y 28}, d_{W 39, S W C N T}$, and $d_{Y 28, S W C N T}$, respectively. Corresponding to the adsorption of the SWCNT on the YAP65 WW, there is a drop of $d_{W 39, S W C N T}$ and $d_{Y 28, S W C N T}$ in the first ns. And then $d_{W 39, Y 28}, d_{W 39, S W C N T}$, and $d_{Y 28, S W C N T}$ all kept almost as a constant at $\sim 6.0 \AA$ with small fluctuations until $\mathrm{t}=45 \mathrm{~ns}$, showing that the two key residues were "confined" in their native state, and the initial adsorption of the SWCNT does not impact much on their structures and orientations. In the period of $t=45$ - 50 ns, there were an impulse jump in $d_{W 39, Y 28}$ to a value around $9 \AA$ and a drop in $\mathrm{d}_{\text {Y } 28, \text { SWCNT }}$ to a valley around $4 \AA$ A. These changes indicate the detachment of W39 with Y28 and the approach of Y28 to the SWCNT. However, $\mathrm{d}_{\text {W39,SWCNT }}$ decreased sharply to $<4 \AA$ at the same time and remained nearly unchanged for the rest of the simulation times, which indicates a strong and favorable interaction between W39 and the SWCNT. It is interesting to note that this favorable tryptophan-CNT interaction has also been observed in recent experiments for CNT-peptide interactions $[33,34]$. Therefore, even though we do not have direct experimental evidence at this point for this plugging of SWCNTs, it might not be totally impossible due to the strong and favorable interactions between CNTs and hydrophobic residues with aromatic rings such as tryptophan residues. It was very interesting to find that both $d_{Y 28, W 39}$ and $d_{Y 28, S W C N T}$ returned to their previous values (before $\mathrm{t}=45 \mathrm{~ns}$ ) at $\sim 50 \mathrm{~ns}$. A careful examination of the trajectory movie showed that there was a rotation of the SWCNT orientation, with the SWCNT firmly seizing the W39 in the period of $\mathrm{t}=50-70 \mathrm{~ns}$. After $\mathrm{t}=70 \mathrm{~ns}, \mathrm{~d}_{\mathrm{Y} 28, \mathrm{SWCNT}}$ decreased to a small value again $(\sim 4 \AA)$, and the $\mathrm{d}_{\mathrm{W} 39, \mathrm{Y} 28}$ turned to a significantly larger value (8-12 $\AA$ ). This indicates a significantly larger separation of the two active-site residues, which corresponds to a wide opening of the second and third $\beta$-strands, indicating a potential loss of its function.

\section{Stability and hydrophobic interaction}

We had computed various interaction energies of the system as functions of the interface area between YAP65 WW domain and SWCNT (shown in Fig. 3). For the interaction energy of the total system (using the initial state as reference, see Fig. 3(a)), there is a basin at about $\mathrm{S}=280 \AA^{2}$, with the interaction energy about $30.0 \mathrm{kcal} / \mathrm{mol}$ lower than the reference state, the state where YAP65 WW domain and the SWCNT are well separated. From Fig. 2(b), we know that $S \sim 280 \AA^{2}$ corresponds to the state in which SWCNT plugs into the YAP65 WW domain. Therefore, the complex with SWCNT inserted into the core of YAP65 WW domain is the energetically more favored state. 

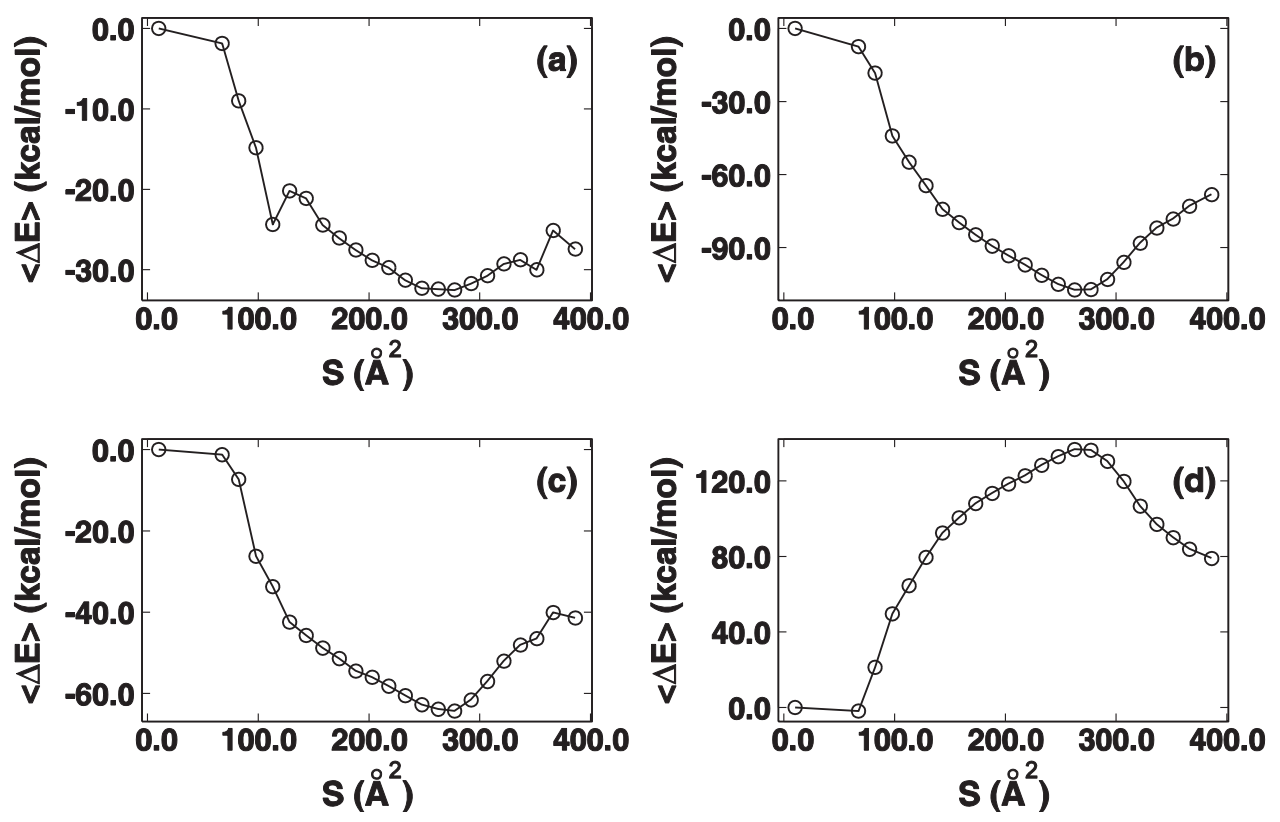

Fig. 3. Interaction energy from the initial state (a) of the total system, (b) of solutes, (c) of solvent, and (d) between solute and solvent, as a function of the interface area $S$ between the YAP65 WW domain and the SWCNT, averaged from the six trajectories of the MD simulations. The interaction energy of the initial state, with the SWCNT and WW domain well separated, is set as 0 .

Fig. 3(b-d) show the interaction energies of the solute (YAP65 WW domain and SWCNT), the solvent (the water molecules), and between solute and solvent, as functions of the interface area, respectively. As shown in these figures, the internal interaction energies of the solute and the solvent (see fig. 3(b and c)) change in the opposite direction of the interaction energy between the solute and the solvent (see fig. 3(d)). Clearly, in our current system, the internal interaction energies of the solute and the solvent dominate the total energy of the system, which both have an energetic basin around $S=280 \AA^{2}$. This implies that the solute and the solvent (water) are favored to interact with themselves and not favored to interact with each other, i.e., a separation of the two is energetically more favored, which is best described as so-called hydrophobic interactions. It is the hydrophobic interaction between the YAP65 WW, SWCNT and water that makes the plugging of SWCNT into YAP65 WW domain.

\section{Binding site and structural flexibility}

In each case of a SWCNT plugging into the YAP65 WW domain, we have found that the YAP65 WW domain displays the same binding site after relentless search. It was shown above that the hydrophobic interaction dominated the binding and plugging of the SWCNT. However, there are also hydrophobic residues, including a tryptophan residue, on the first $\beta$-strand of the YAP65 WW. Thus the question was why the SWCNT plugged 
into the gap between the second and third $\beta$-strand instead of that between the first and second $\beta$-strand. The flexibility of different part of the YAP65 WW domain may be a factor of selection of the plugging site. To illustrate this idea, Fig. 4 shows the root mean square fluctuations (RMSF) of the average of non-hydrogen atoms of each residue for the YAP65 WW domain (the simulation was performed without any SWCNT or ligand). Generally, the loops and turns have larger fluctuations. The notable point of this figure is the difference between the three $\beta$-strands. Clearly, the fluctuations of the third $\beta$-strand residues are significantly higher than those of the other two $\beta$-strands. This indicated that the third $\beta$-strand was more flexible than the other two $\beta$-strands. That is, the gap between the second and third $\beta$-strand is easier to be opened, and thus the SWCNT is easier to penetrate it.

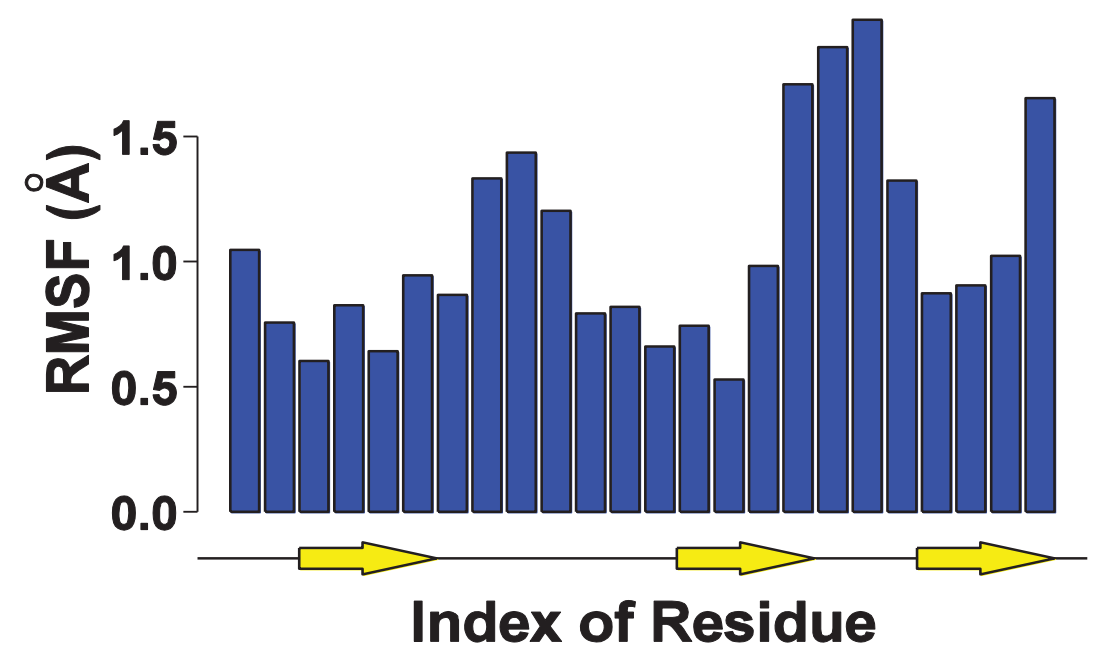

Fig. 4. RMSF of the non-hydrogen atoms for the simulation of the YAP65 WW domain without SWCNT. The yellow arrows show the three $\beta$-strands of the WW domain. The secondary structure information of the domain is obtained by DSSP [74].

\section{Effect on protein function}

As shown above, the SWCNT plugged into the second and third $\beta$-stands and formed a stable complex. In the protein-SWCNT complex, two active residues of YAP65 WW domain (W39 and Y28) are separated, thus the primary functional unit, the scaffold, for binding with prolines in the proline rich motifs (PRM) is disrupted. In general, the SWCNT insertion of the binding site is much more disruptive than the typical surface adsorption, which may result in the loss of the original function of the WW domain. To demonstrate this, we performed simulations for the system with an additional PRM ligand (sequence GTPPPPYTVG), which binds to both the YAP65 WW domain in its native state [71], and to the protein-SWCNT complex. In the PRM binding simulations, the PRM was initially placed at $25 \AA$ away from the center of the protein-SWCNT complex. There were 7045 TIP3P water molecules and one $\mathrm{Cl}^{-}$(which was used to neutralize the system), and all other MD setups and parameters were similar to the previous simulations. Ten independent runs were performed for each system in NVT ensemble at $298 \mathrm{~K}$. 

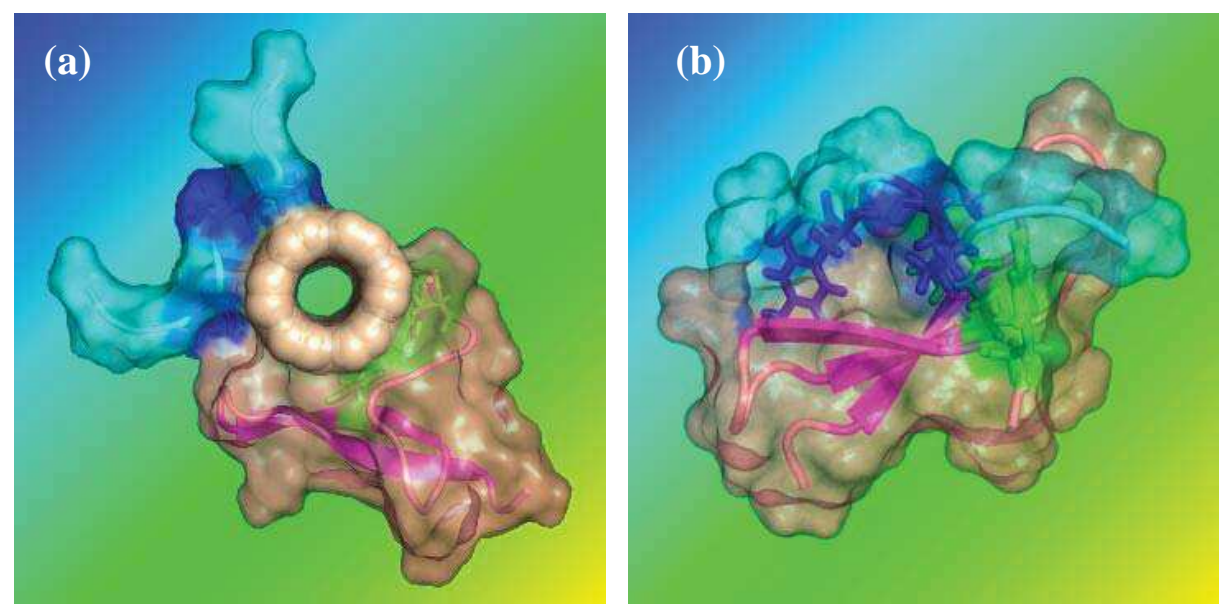

Fig. 5. A typical structure of (a) the protein-SWCNT complex with the adsorbed proline-rich motif (PRM) and (b) the domain binding with the PRM (PDB code: 1JMQ). Here the YAP65 WW protein domain is shown in cartoon with magenta strand and salmon loop, and the key residues (W39 and Y28) are identified by green sticks. The atoms of the $(6,6)$ armchair SWCNT are shown in wheat spheres. The PRM ligand with the sequence GTPPPPYTVG is shown in cyan cartoon with the key residues identified by blue sticks. The solvated surfaces of the complex are shown in semitransparent.

Our simulations showed that the protein-SWNT complex was stable, and the PRM always bound to the SWCNT instead of the binding site of the YAP65 WW, as shown in one representative structure in Fig. 5(a). As for comparison, we also showed the native structure with the PRM in Fig. 5(b). Clearly, the binding site (labeled by the green stick in these two figures) had been blocked by the SWCNT in the YAP65 WW domain-SWCNT complex. These findings indicate that the insertion of the SWCNT disrupted and blocked the binding site of the YAP65 WW domain, and disabled its function of identifying and binding with the PRM. To validate this prediction from our in silico approach, in vitro and/or in vivo experiments are highly needed. Interestingly, there is recent evidence from both experiment and theory showing that some antibody from mouse immune repertoire can "absorb" the C60 nanoparticle through similar hydrophobic interactions [75, 76].

\subsection{Competitive binding with ligands to receptors}

The scheme of protein functioning can be roughly described into three steps: binding, transforming, and releasing. The binding, especially the specific binding, between proteins and ligands is the primary step for the protein functioning. Again, hydrophobic interactions often play a vital role in the binding of proteins with ligands. If pristine CNTs (which are extremely hydrophobic) pass through cell membranes and possess stronger binding affinity than ligands with hydrophobic binding sites of some key proteins, these CNTs may hold their hydrophobic binding sites, obstruct their binding processes, and disrupt the functions of these proteins. This competitive binding may be another potential mechanism of toxicity of hydrophobic nanoparticles, including CNTs. All the aforementioned studies focus on the direct interactions of nanoparticles with proteins. The effect of nanoscale particles on the ligand-receptor binding, i.e., a three way nanoparticle-ligand-protein competitive binding, is much less studied. 
We take a proline-rich ligand (PPPVPPRR) and its binding module, the SH3 domain, together with a pristine SWCNT as an example to illustrate the idea. The SH3 domain is one of the most abundant protein interaction domains [77], and usually found in the signaling and regulatory proteins as the functional module to identify and bind PRM of their binding partners [78-84]. Fig. 6(a) shows the native structure of the $\mathrm{SH} 3$ domain binding with the ligand. It exists as a characteristic $\beta$-barrel fold which consists of five $\beta$-strands arranged as two tightly packed anti-parallel $\beta$-sheets [85]. The residues, including F8, W36, P50, and Y53, are the key residues to bind ligands $[78,86]$; and the hydrophobic interaction is the dominant force for the binding process [87]. The SWCNTs are armchair of $(m, n)$, where $m=n=3$, corresponding to the tube diameters of $4.04 \AA$ with a length of $19.54 \AA$. The ligand, SWCNT and protein are initially well separated from each other. The initial distances of the geometric centers, of both the SWCNT and the ligand, from that of the $\mathrm{SH} 3$ domain are set at $30 \AA$, but in different orientations. The distance between the geometric centers of the SWCNT and ligand is about $42 \AA$. They were solvated with TIP3P model water as well. We performed 20 independent simulations for the SH3 domain, the PRM ligand, and the SWCNT with different initial velocities, each with 200 ns (see more detail in Computational Methods).

\section{Interaction of SH3 domain, ligand and SWCNT}

We find that the SWCNT occupies the binding pocket and contacts directly with the key residues of the $\mathrm{SH} 3$ domain in 13 of the 20 simulation trajectories. In this way, the SWCNT prevents the ligand from forming the native contacts with the $\mathrm{SH} 3$ domain binding pocket.
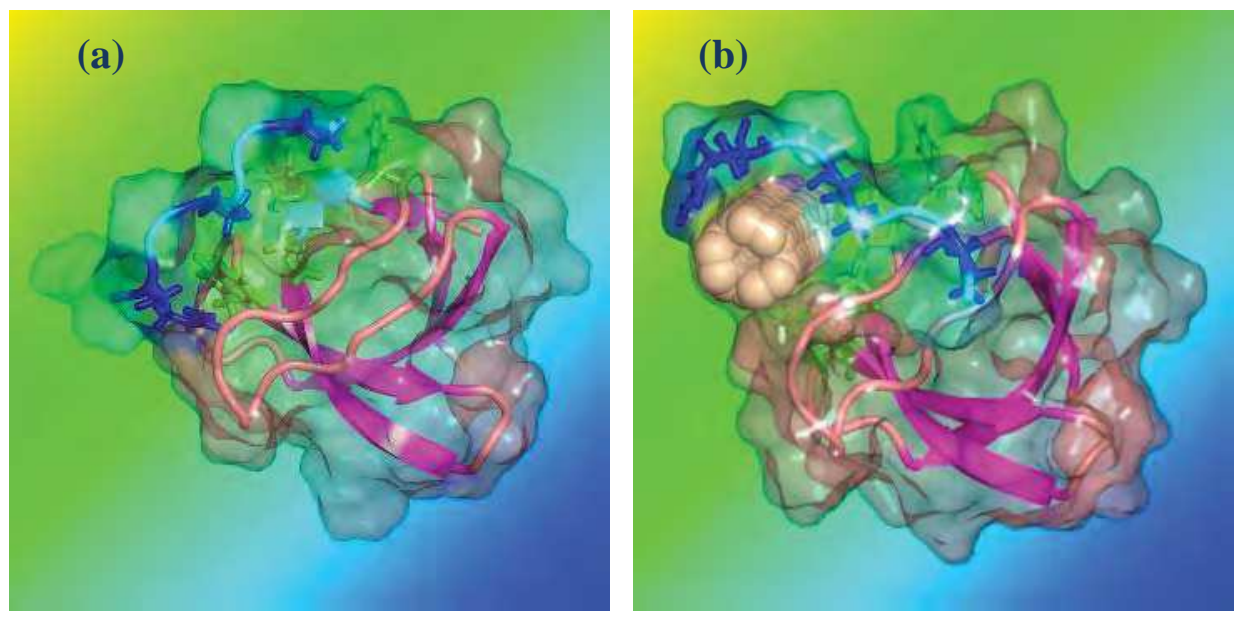

Fig. 6. Structures of (a) the SH3 domain binding with the PRM ligand (PDB: 1CKB) in its native state; and (b) a typical snapshot in which the SWCNT occupies the binding pocket and contacts with the key residues of the $\mathrm{SH} 3$ domain, while the ligand binds partly to the SWCNT and partly to the SH3 domain; and Here the SH3 domains are shown in cartoon with magenta strand and salmon loop, and the key residues (F8, W36, P50 and Y53) in the pocket are identified by green sticks. The atoms of the SWCNT are shown in wheat spheres. The ligands with sequence PPPVPPRR are shown in cyan cartoon with the key residues identified by blue sticks. The solvated surface of complexes is shown semitransparent. 
Fig. 6(b) shows a typical structure for the case that SWCNT occupies the binding pocket of SH3 and the ligand binds to the SWCNT. The SWCNT directly contacts with nine residues of the $\mathrm{SH} 3$ domain in the pocket, including the key residues W36, P50, and Y53. In the other 12 cases, the SWCNT contacts with at least one of the key residues of the binding pocket of the SH3 domain. And in 11 of those 13 trajectories, the ligand binds partly to the SWCNT and partly to the surface of the SH3 domain (near the SWCNT). In other two cases, the ligand only binds to the surface of the SH3 domain (but not the active site) and does not bind to the SWCNT. Even though the other seven trajectories do not show a direct binding of the SWCNT with the SH3 domain binding pocket, the SWCNT does display some tendency of the binding. For example, in three of the seven trajectories, the SWCNT is getting close to the key W37 residue in the binding pocket; and in the other two trajectories, it is getting close to the key Y03 residue. The incomplete binding might be caused by the fact that our simulation lengths, 200+ ns, are still not long enough.

The binding process is illustrated by representative snapshots as well as the interface areas between the SH3 domain, the ligand, and the SWCNT, using a typical trajectory (see Fig. 7), in which the SWCNT first binds onto the SH3 and then the ligand binds onto the SWCNT.
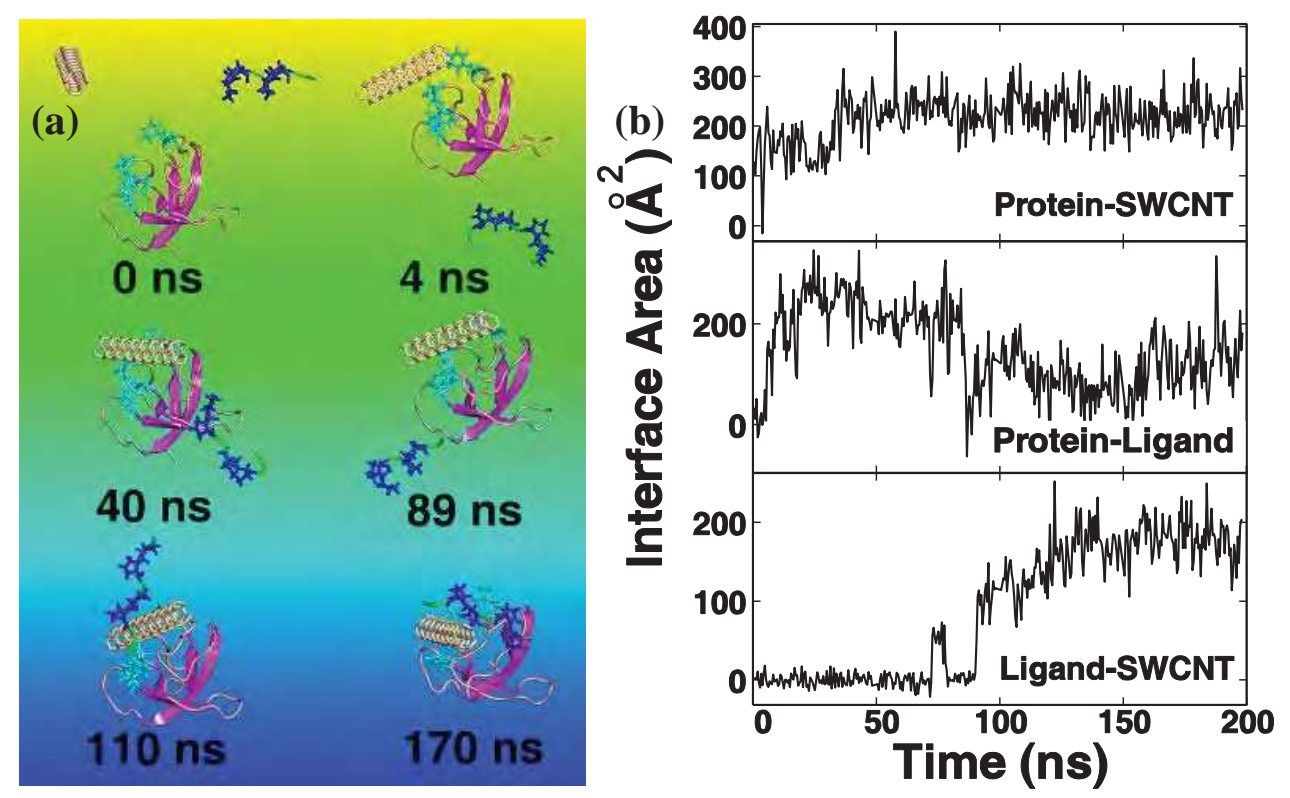

Fig. 7. One representative trajectory in which the SWCNT adsorbs onto the binding pocket and makes direct contacts with the key residues of the $\mathrm{SH} 3$ domain, while the ligand binds partly with the SWCNT and partly with the SH3 domain. (a) Some representative snapshots. Here the proteins are shown in cartoons with magenta strand and salmon loop. The key residues of the protein domain are noted by red sticks, the ligands with the sequence PPPVPPRR are shown in cyan cartoon with the key residues identified by blue sticks. The SWCNTs are shown in wheat sticks. (b) The protein-SWCNT, protein-ligand, and ligandSWCNT interface areas as function of time. 
To illustrate the binding process of the ligand, we also show the interface areas of the ligand with the SH3 domain (protein-ligand), and the ligand with the SWCNT (ligand-SWCNT), denoted by $S_{P L}$ and $S_{L C}$, respectively (see Fig. $7(b)$ ). At $t=0, S_{P L}=S_{L C}=0$ since the ligand is separated from both the SH3 domain and the SWCNT initially. In the first $5 \mathrm{~ns}$, S $\mathrm{PL}$ increases to about $200 \AA^{2}$ very quickly, and maintains that value until $\mathrm{t}=\sim 90 \mathrm{~ns}$. At the snapshot of $\mathrm{t}=40 \mathrm{~ns}$, the ligand is adsorbed onto the SH3 domain (but not at the binding pocket). On the other hand, $S_{\text {LC }}$ still keeps at about 0 . The most notable event happened at about 90 ns. After that, $\mathrm{S}_{\mathrm{PL}}$ decreases quickly from $200 \AA^{2}$ to a very small value, and later recovers back to $\sim 150 \AA^{2}$. Meanwhile, $S_{\text {LC }}$ increases to about $100 \AA^{2}$. At $t=89$ ns, the ligand has temporarily separated from the SH3 domain, diffuses to the other side of the SH3 domain, and then binds to both the SWCNT and the SH3 domain (the structure is shown in the snapshot at $100 \mathrm{~ns}$ ). From $t=90 \mathrm{~ns}$ to $t=200 \mathrm{~ns}$, both $\mathrm{S}_{\mathrm{PL}}$ and $\mathrm{S}_{\mathrm{LC}}$ remain at these values with normal fluctuations, indicating a stable three-way bound structure among the SWCNT, the ligand, and the $\mathrm{SH} 3$ domain.

From the interface areas, we showed the main picture of the binding process of the SWCNT, the ligand and the $\mathrm{SH} 3$ domain. To give a more detailed picture about the binding process at the residue level, we had computed the contact number of the SWCNT and the ligand with the residues of $\mathrm{SH} 3$ domain. Here we used the protein residue as a unit base in accounting (see Fig. 8), and a contact is counted if the distance between a non-hydrogen atom in the

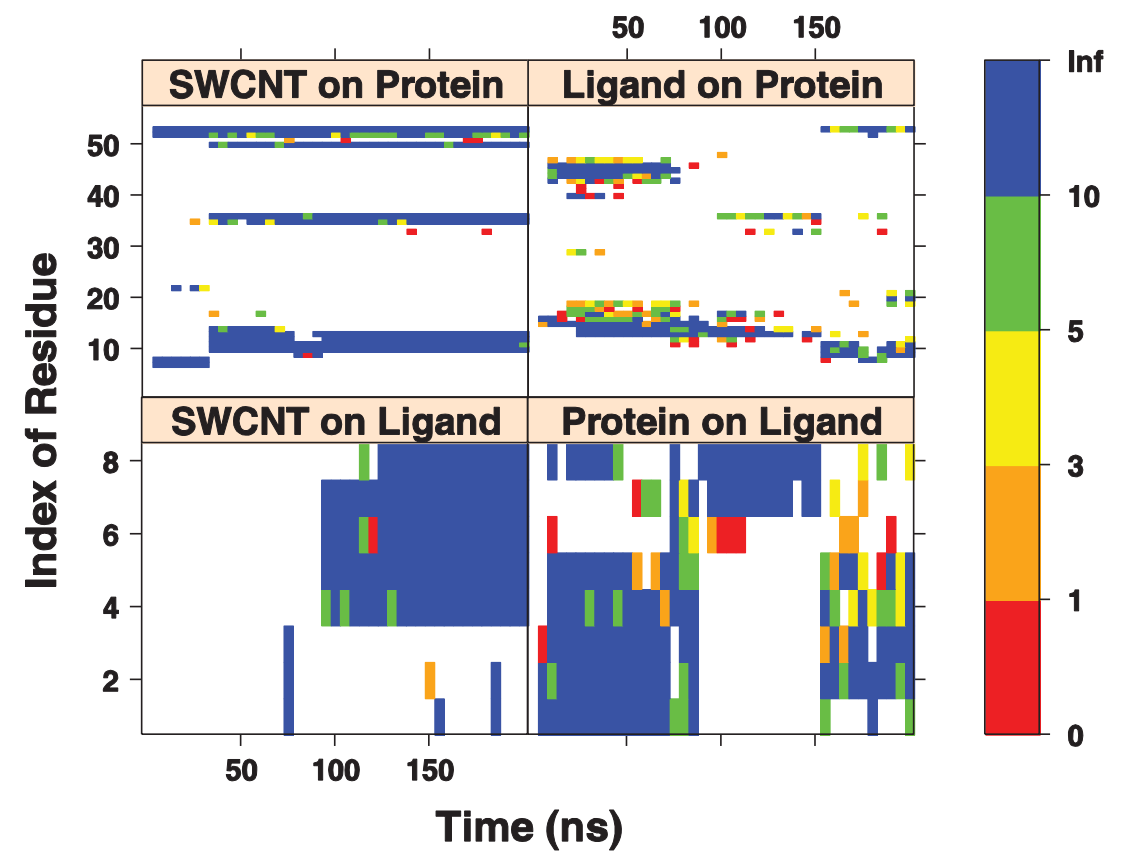

Fig. 8. Numbers of the contacts of the SWCNT and the ligand with the residues of SH3 domain, together with those of the SH3 domain and the SWCNT with the residues of the ligand. The number denoted by the color and the " blank" meaning no contact. There is a contact if the distance between a non-hydrogen atom in the bound object and a nonhydrogen atom in the residue is less than $5 \AA$. 
bound object and a non-hydrogen atom in a residue is less than $5 \AA$. Generally speaking, the closer the object is to the protein residue, the larger the contact number is between the protein and the object, and the stronger the object binds to the protein residue. Similarly, from the contacts of the SWCNT on the SH3 domain, we found that there were three stages in the binding process. In the initial stage, from 0 to $\sim 3 \mathrm{~ns}$, the SH3 domain and the SWCNT were separated; in the second stage, from $\sim 3$ to $\sim 35 \mathrm{~ns}$, the SWCNT was adsorbed onto four residues of the $\mathrm{SH} 3$ domain; in the third stage, from $\sim 35 \mathrm{~ns}$ to the end of the simulation, the SWCNT bound closely with many residues (up to 9) of the SH3 domain, including the key residues $\mathrm{W} 36, \mathrm{P} 50$, and $\mathrm{Y} 53$.

Furthermore, the contacts between the SWCNT and the ligand are found to be quite stable, i.e., these contacts remain in contact once they appear (see Fig. 8). On the contrary, the contacts between the ligand and the protein (both ligand on protein and protein on ligand) vary with time, indicating that the relative positions between the ligand and the $\mathrm{SH} 3$ domain change with time much more often. Further, the great changes of the ligand-protein contacts happen at $\mathrm{t}=\sim 88 \mathrm{~ns}$ (Fig. 8), corresponding to the previous observation that the ligand temporarily separates from the $\mathrm{SH} 3$ domain, then diffuses to the other side of the SH3 domain, and finally re-adsorbs onto both the SWCNT and the SH3 domain. Conformational fluctuations of the ligand on the SWCNT and SH3 domain are observed after the final adsorption. For example, from 90 ns to $150 \mathrm{~ns}$, the ligand binds both the SH3 domain (on the residues 12-13, 16, and 36) and the SWCNT by the C-terminal residues; while from 150 ns to $200 \mathrm{~ns}$, the ligand binds the SH3 domain (on the residues 9 11, and 53) by the N-terminal and the SWCNT by C-terminal (see also Fig. 8).

\section{Free energy landscape}

Fig. 9(a) shows the free energy landscape as a function of two reaction coordinates: the interface area between the SH3 domain and the SWCNT ( $\mathrm{SPC}$ ), and the minimal distance between the SWCNT and the key residues of the SH3 domain (including F08, W36, P50, and Y53), denoted by $\mathrm{D}_{\mathrm{KC}}$. Clearly, the SWCNT will prevent the regular binding of the ligand to the $\mathrm{SH} 3$ domain if it binds to one of the key residues. Thus, the reaction coordinate $\mathrm{D}_{\mathrm{KC}}$ could be a good reaction coordinate to describe the binding free energy landscape. To obtain a reasonable result, we sampled the structures with a time interval of 20 ps in the last $180 \mathrm{~ns}$ of all the 20 trajectories. The global minimum in this landscape is found at $\mathrm{S}_{\mathrm{PC}} \sim 220 \AA^{2}$ and $\mathrm{D}_{\mathrm{KC}}<5.0 \AA$ (see Fig. 9(a)) with a binding free energy of $-6.08 \mathrm{kcal} / \mathrm{mol}$, which corresponds
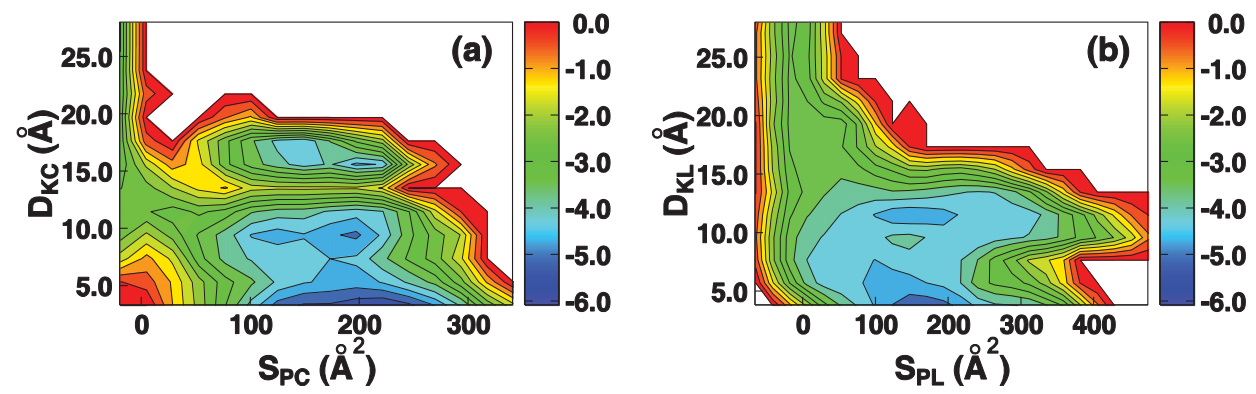

Fig. 9. The binding free energy landscapes of the SWCNT (a) and the ligand (b) with the SH3 domain. "S $\mathrm{SC}_{\mathrm{PC}}$ and "S $\mathrm{S}_{\mathrm{PL}}$ " denote the interface area of the SWCNT and ligand with the SH3 domain, respectively. " $\mathrm{D}_{\mathrm{KC}}$ " and " $\mathrm{D}_{\mathrm{KL}}$ " are the minimal distance of the SWCNT and the ligand from these key residues, respectively. The unit of the free energy is $\mathrm{kcal} / \mathrm{mol}$. 
to the state where the SWCNT binds to the SH3 domain binding pocket. Two local minima, in which $\mathrm{S}_{\mathrm{PC}} \sim 150 \AA^{2} \& \mathrm{D}_{\mathrm{KC}} \sim 8.0 \AA$, and $\mathrm{S}_{\mathrm{PC}} \sim 180 \AA^{2} \& \mathrm{D}_{\mathrm{KC}} \sim 18.0 \AA$, are also observed. Detail studies show that these two states correspond to the SWCNT binding to the side (the region around the residue W37) and the opposite (the region around the residue Y03) of the binding pocket of the $\mathrm{SH} 3$ domain, respectively.

The binding of the ligand to the $\mathrm{SH} 3$ domain is also described by the free energy landscape as a function of the two equivalent reaction coordinates: the interface area between the ligand and the $\mathrm{SH} 3$ domain $\left(\mathrm{S}_{\mathrm{PL}}\right)$, and the distance between the ligand and the key residues of the SH3 domain $\left(\mathrm{D}_{\mathrm{KC}}\right)$ (shown in Fig. 9(b)). The lowest binding affinity for the ligand is $-5.51 \mathrm{kcal} / \mathrm{mol}$, which is $0.57 \mathrm{kcal} / \mathrm{mol}$ less than the SWCNT shown above. This explains why the PRM ligand loses the competition to the SWCNT in the binding to the SH3 domain. Interestingly, the binding free energy landscape also shows that there are two basins, with the free energy of $-5.51 \mathrm{kcal} / \mathrm{mol}$ (the lowest for the ligand binding) and $-5.16 \mathrm{kcal} / \mathrm{mol}$, respectively. Both states have similar values of $\mathrm{S}_{\mathrm{PL}} \sim 180 \AA 2$, i.e., adsorbing onto the SH3 domain with similar contacting surface areas. The difference lies in the $\mathrm{D}_{\mathrm{KL}}$, i.e., the distance to the key residues of the $\mathrm{SH} 3$ domain binding pocket. The deeper one has a very small value of $\mathrm{D}_{\mathrm{KL}} \sim 5.0 \AA$, which indicates that the ligand is around the binding pocket. The other basin has a larger value of $\mathrm{D}_{\mathrm{KL}} \sim 12.0 \AA$, indicating that the ligand is far away the binding pocket. We note that the same $D_{K L}(\sim 12 \AA)$ does not mean that there is only one binding site for the ligand in this case. A detailed study shows that the ligand binds to a broad region of the SH3 domain with residues 11-19, 23-25, and 43-47 involved.

\section{$\pi-\pi$ stacking interaction}

From Fig. 9(a), there are three basins in the free energy landscape of the SWCNT binding on the SWCNT. When the SWCNT was in the global minimum (binding on the binding pocket), it contacted with at least one of the aromatic residues, including F08, W36 and Y53. The upper panel of Fig. 10 shows the typical binding mode of the SWCNT contacting with these three aromatic residues, in which their side chains of are almost parallel with the SWCNT. For the two local minima, the SWCNT contacted with the Y03 and W37, respectively (shown in the lower panel of Fig. 10). Overall, the SWCNT always contacts with aromatic residues when it binds onto the $\mathrm{SH} 3$ domain. In the $\mathrm{SH} 3$ domain native structure, there are a total of 47 residues exposed to environment (solvent, ligand, or SWCNT), but only ten of them are hydrophobic and five of them aromatic residues. This indicates the SWCNT is favored to contact with the hydrophobic and aromatic residues (more below).

In order to describe quantitatively the degree by which the SWCNT favors to contact with the hydrophobic and aromatic residues, we estimated the p-values of distribution of the hydrophobic and aromatic residues in contact with the SWCNT based on the $X^{2}$ hypothesis [88] (see Fig. 11). It was found that the SWCNT is remarkably favored in binding with the hydrophobic residues of the SH3 domain. The p-value for the SWCNT in contacting with hydrophobic residues (Ile, Val, Leu, Met, Ala, Trp, and Phe, following the hydrophobicity classifications of this NCBI website: http://www.ncbi.nlm.nih.gov/Class/Structure/aa/aa_explorer.cgi), aromatic residues (His, Tyr, Phe, and Trp), and the hydrophobic aromatic residues (Phe and Trp; here we do not include Tyr residue, even though many people might classify Tyr to be hydrophobic aromatic residue as well) were $6.51 \times 10^{-3}, 2.42 \times 10^{-12}$, and $2.00 \times 10^{-19}$, respectively. This indicates that the interactions between the SWCNT and the hydrophobic residues, particularly the aromatic residues ( $\pi-\pi$ stacking interactions), play an important role in 
the binding of SWCNT and proteins. We note that favorable interactions of the carbon nanotube with the hydrophobic and aromatic residues had also been observed in recent carbon nanotube-peptide experiments [33, 34, 89]. That is, these interactions are independent of the sizes of proteins and CNTs. Therefore, even though the SWCNT used in our simulation is quite small, the observation of the high probability to occupy the binding pocket of the protein by the SWCNT is extendable to the hydrophobic nanoscale particles of larger lengths, which is expected to be observed with more extensive computations with the development of the supercomputers as well as advanced simulation techniques. It should also be noted that the $\pi-\pi$ stacking interactions might be underestimated in standard force fields due to the lack of polarization, and more advanced techniques such as quantum mechanics simulations might be needed to fully catch the effect; however, a recent study shows that classical simulations can be sometimes even better to capture this $\pi-\pi$ stacking interactions than the quantum mechanics simulations due to the facts that the classical force fields are often directly fitting from experimental data, while the quantum mechanics simulations, on the other hand, might suffer from the limited size and boundary conditions [90].
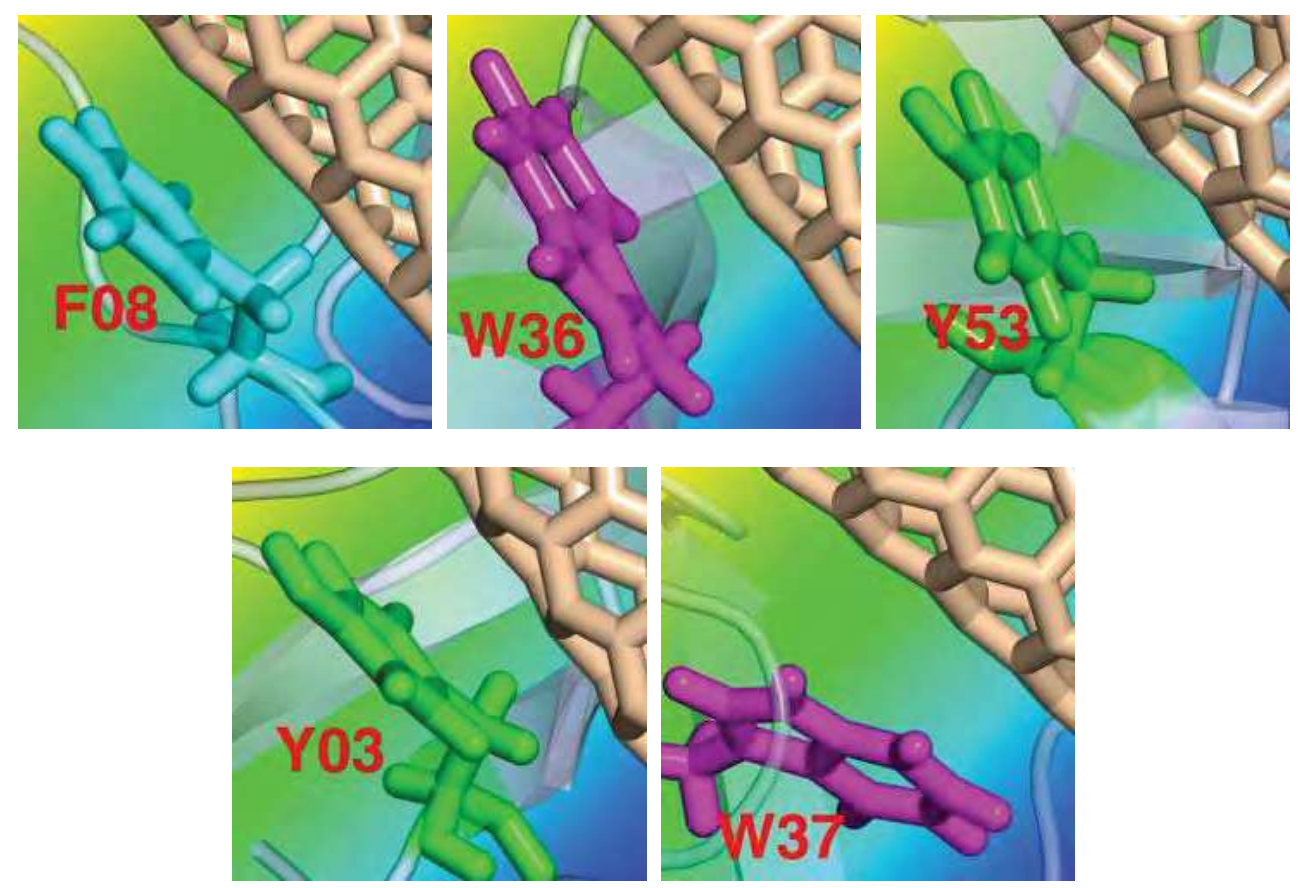

Fig. 10. Interaction between the SWCNT and the aromatic residues of SH3 domain. The top panel shows the key residues of the binding pocket, including F08, W36 and Y53. The bottom panel shows the aromatic residues which are exposed but not belong to the binding pocket, including Y03 and W37. Here the SH3 domains are shown in semitransparent sky blue cartoon. The aromatic residues are shown in sticks (magenta tryptophan, cyan phenylalanine, and green tyrosine) and labeled by red text. The SWCNTs are shown in wheat sticks. 


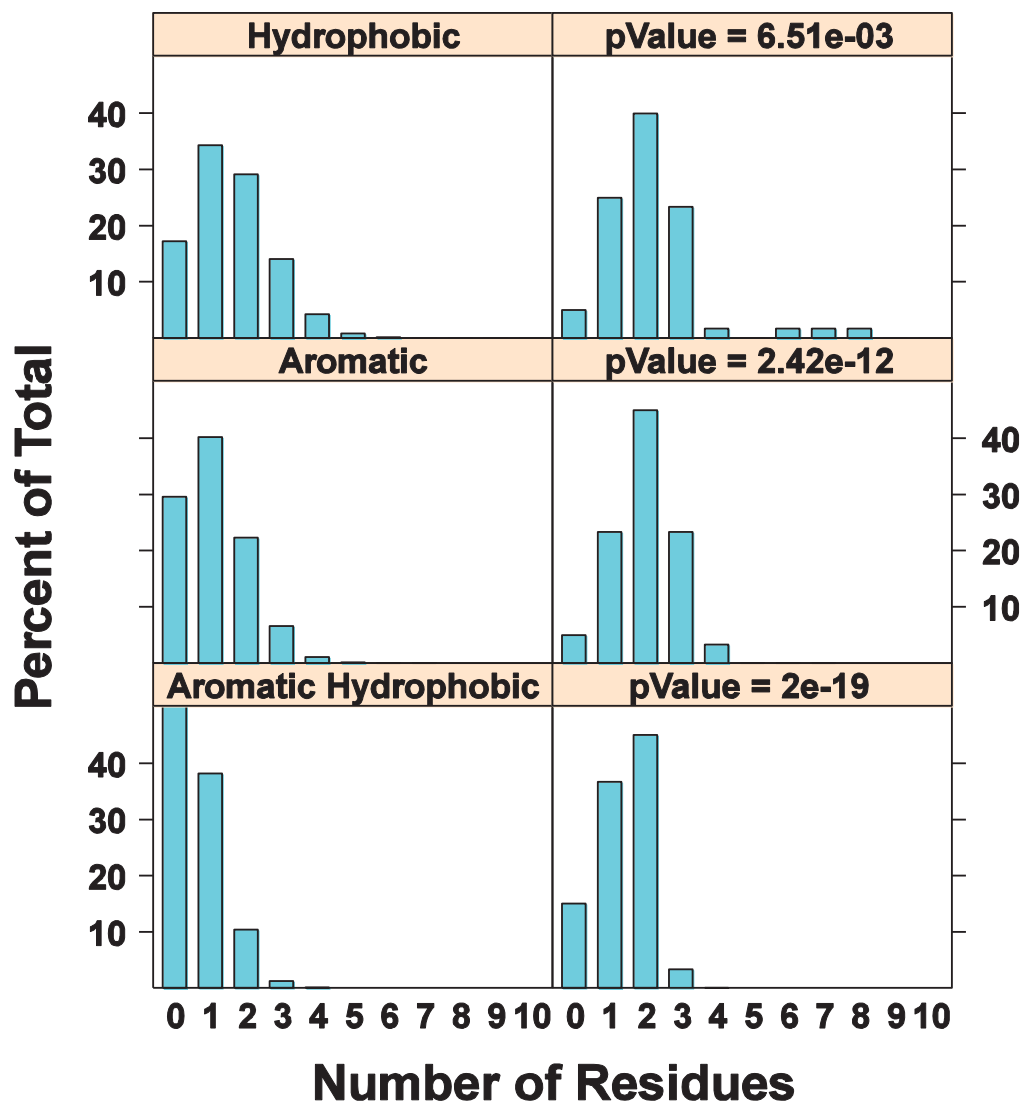

Fig. 11. Distribution of the number of the hydrophobic, aromatic, and aromatic hydrophobic residues contacted with SWCNT. Here a residue is contacted with the SWCNT when the distance between one of its non-hydrogen atoms and SWCNT is less than $5 \AA$. The left column shows the expected distribution based on the number of the residues and the total number of the residues contacted with SWCNT. The right column shows the practical distribution. The p-values for the distribution are estimated on the $\chi^{2}$ hypothesis test, and shown on the right column.

\section{Effect on the binding event}

It is noteworthy that a previous MD simulation has shown that the ligand could recognize the binding pocket and form the native binding mode with the SH3 domain, in the absence of nanoscale particles [87]. The long-range electrostatic interactions are found to play an important role in the ligand recognition of the binding pocket, through its two arginine residues on the C-terminal [87]. The PRM ligand's R7 and R8 residues have favorable longrange electrostatic interactions with SH3-domain's acidic residues, D14, E16, D17, and E33 on RT and n-sCr loops, near one end of the binding pocket, which provides guidance to an accelerated ligand binding mode search. This electrostatically accelerated association of proteins was also previously observed in experiments [91, 92]. And studies had confirmed 
that the arginine residue is conserved in the PRM to this SH3 domain [78, 79]. The stability of the final binding, on the other hand, is driven by the structural match and hydrophobic interactions between the ligand (proline residues and V4) and the SH3 domain (F8, F10, W36, P50, P52, and Y53), in addition to the salt-bridges formed by R7/R8 (see Fig. 6(a)). In our current complex system with the presence of SWCNT, the SWCNT wins the competition over the ligand for the binding pocket even without any guidance from the long-range electrostatic interactions (since SWCNT is uncharged in our simulations), indicating a very strong hydrophobic interaction between the SWCNT and the SH3 domain. Interestingly, the ligand still manages to locate the area of the active site and bind with both the SWCNT and SH3 domain (though no longer in the binding pocket), which implies the PRM ligand still maintains some recognition capability due to the long-range electrostatic interactions through its two C-terminal arginine residues.

The structural match between the ligand and active site stabilized the native binding mode. However, it raised the demand for an extensive conformational space mapping even with the electrostatic acceleration. And the hydrophilic residues in the ligand (the two terminal arginines) often shield the hydrophobic ones in the water environment to "inhibit" their exposure to the active site. On the other hand, the hydrophobic interactions between the SWCNT and the hydrophobic residues of the binding pocket, particularly those aromatic ones, are strong and nonspecific. This makes the SWCNT more straightforward to be adsorbed onto the binding pocket. Thus it takes only about $35 \mathrm{~ns}$ for the SWCNT to reach a stable binding state at the active site, while it takes $\sim 130 \mathrm{~ns}$ for the ligand to come near the $\mathrm{SH} 3$ domain in the previous MD simulations [87]. This is also consistent with the above free energy landscape analysis, which reveals that the SWCNT is more favorable than the PRM ligand to be bound to the active site of the SH3 domain. That is, in the competition for the binding pocket of the SH3 domain, the SWCNT has advantages over the PRM ligand in both kinetics and thermodynamics. The SWCNT essentially occupies the binding pocket of the $\mathrm{SH} 3$ domain and interrupts its native binding with the PRM ligand.

\subsection{Interaction with human blood serum proteins}

All the above simulations were done with relatively short CNTs $(\sim 20 \AA)$ and small proteins (SH3 domain has 57 amino acids and WW 26 amino acids) due to the enormous computational resources needed. In order to see whether longer SWCNTs and larger proteins might exhibit similar behaviors, we have recently performed simulations with IBM BlueGene supercomputers on much larger systems - the SWCNTs interacting with human blood serum proteins BSA (bovine serum albumin), $\gamma$-Ig (gamma-globulin), Tf (transferring), and BFg (fibrinogen), also in an attempt to compare with experiments directly. The total number of atoms in the solvated systems is up to 1.06 million. Figure 12 shows one example of the system setup with BFg dimmer interacting with SWCNT in solution. Since this is still an ongoing research, only a very brief description of the current results will be given. More simulation results and detailed comparisons with experiments will be reported elsewhere [93].

To elucidate the mechanism of protein adsorption by the SWCNT, up to 150 ns molecular dynamics simulations have been performed for each protein-SWCNT complex in explicit solvent. In our experiments, including AFM images, fluorescence spectroscopy, and SDSPAGE, we found that these serum proteins display a competitive binding/adsorption with the SWCNTs, with BFg showing the largest adsorption capacity. In addition, the adsorption capacity displays a general trend of $\mathrm{BFg}>\gamma$-Ig $>$ Tf $>$ BSA [93]. 


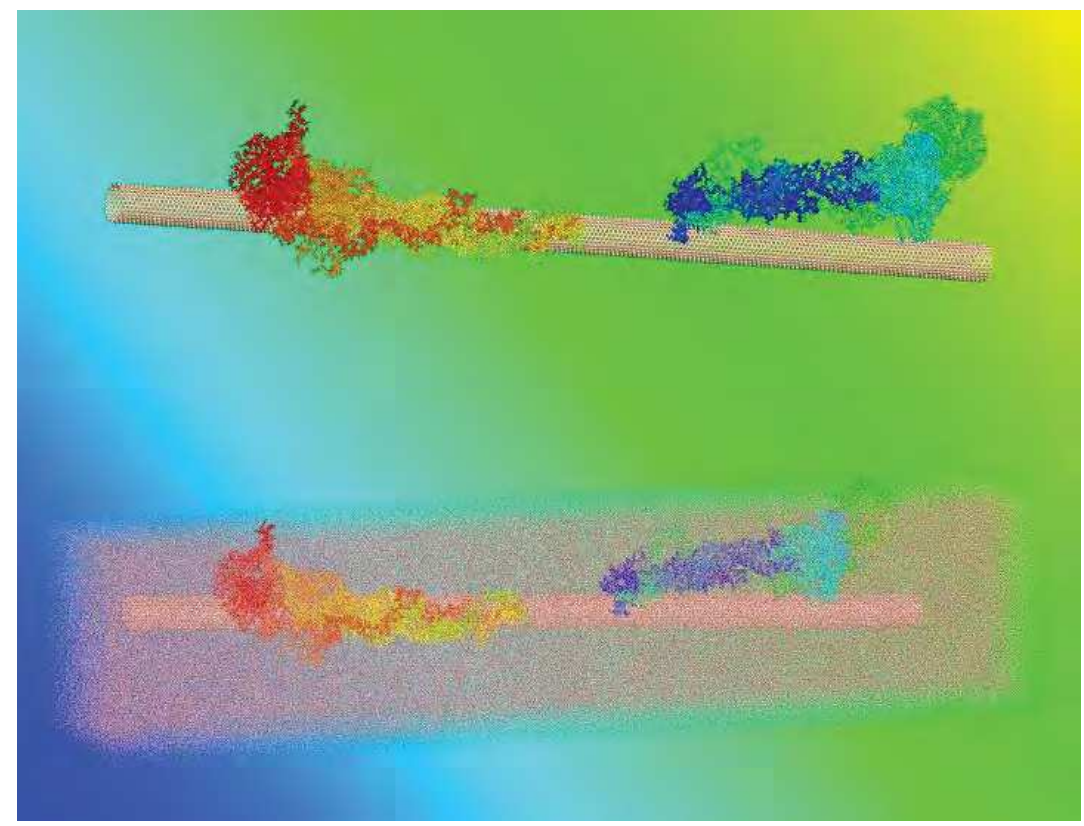

Fig. 12. Representative snapshot from the simulation trajectory of the human blood serum protein BFg interacting with a SWCNT. The solvated complex system has 1,058,598 atoms. The top panel shows the BFg-SWCNT complex alone, while the bottom panel also includes the solvation box. Here the protein BFg is shown in ribbons colored by chain. The SWCNT is shown in wheat sticks, and water in dots (with only $\mathrm{O}$ atoms shown).

As mentioned above, the aromatic residues, tryptophan (Trp), tyrosine (Tyr) and phenylalanine (Phe), could produce $\Pi-\Pi$ stacking interactions between aromatic rings of amino acids and six-member rings of the SWCNT. As a first analysis, the numbers of these three kinds of amino-acid residues on the surface of initial conformation for each protein molecule were counted. The approximate numbers of (Trp, Tyr, Phe) for BFg, $\gamma$ - $\mathrm{Ig}$, Tf and BSA were in sequence of $(14,47,27) ;(2,25,10) ;(1,13,15)$; and $(1,9,10)$; respectively. This implies that the aromatic residues on the surface of these blood serum proteins particularly consist of Tyr and Phe, and lack of Trp, except for BFg which has 14 surface Trp residues. Most of the Trp residues are wrapped in the core of proteins, and play a lesser role in these serum protein-SWCNT complex adsorptions, which seems to be different from the above WW domain and $\mathrm{SH} 3$ domain cases where Trp residues play a significant role.

The snapshots of MD simulation results describe the preferred binding sites on proteins for SWCNT. If we highlight the residues within $5 \AA$ distance from the surface of SWCNT, the aromatic residues Tyr and Phe stand out as the key residues involved in the adsorption. Interestingly, only Tyr and Phe were observed in the adsorption on the surface of SWCNT (at least in the first 150 ns simulations, longer runs might reveal larger protein conformational changes and exposure of Trp residues to the SWCNT surface as well), and Trp residues made little contribution for adsorption in the present MD simulations, which are still very short in time scale as compared to experiments ( 5 min to 1 hour). Nevertheless, our simulations show that there is a positive correlation between the total numbers of Trp, 
Tyr, and Phe residues and the binding surface areas, thus the adsorption content, of the proteins, respectively.

In order to illuminate the above positive correlation relationship more clearly, we calculated the contact residue number and contact surface area of various proteins, in adsorption of SWCNT versus time. We used the average contact residue number (ACRN) of the protein to quantify its adsorption ability with SWCNT. The ACRN for BFg during 140 to $150 \mathrm{~ns}$ is 94 . The ACRN for $\gamma$-Ig after its saturation during 70 to $150 \mathrm{~ns}$ is 28 . The ACRN for Tf after its saturation during 80 to $150 \mathrm{~ns}$ is 13. The ACRN for BSA after its saturation during 65 to 150 ns is 12. The relationship of ACRN of various protein complex systems shows an order of $\mathrm{BFg}>\gamma$-Ig $>\mathrm{Tf}>\mathrm{BSA}$, which is in good agreement with the experimental findings [93] (and also compatible with the above simple analysis using aromatic hydrophobic residues). Similarly, we computed the average contact surface area (ACSA) for these blood serum proteins (see Fig. 13). The ACSA for BFg, $\mathrm{Y}-\mathrm{Ig}$, Tf and BSA during the responding time ranges are $4360 \AA^{2}, 1240 \AA^{2}, 580 \AA^{2}$ and $470 \AA^{2}$ respectively, which again shows an order of $\mathrm{BFg}>\gamma-\mathrm{Ig}>\mathrm{Tf}>\mathrm{BSA}$, in good agreement with the experimental findings [93].

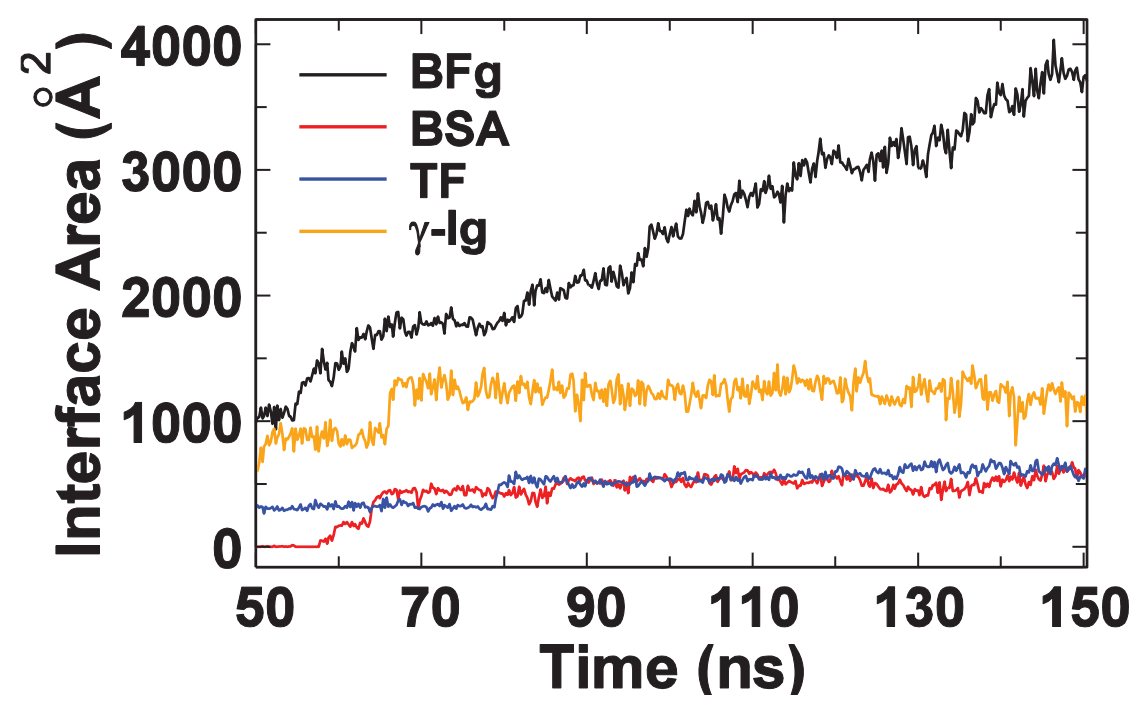

Fig. 13. The protein-SWCNT interface area as a function of time for various human blood serum proteins. Here the interface area is defined as half of the difference between the solvent accessible surface area of the complex and the sum of solvent accessible surface areas of the protein and the SWCNT.

The most abundant Tyr and Phe residues on the surface of BFg molecule can be attributed to the highest protein quantities binding to SWCNT. Interestingly, the $\pi$-cation interactions are also found to play a role, though smaller, in the BFg-SWCNT binding. The average Arg and Lys residues in contact with SWCNT (ACRN-Arg, ACRN-Lys) are found to be about 1.3 and $\sim 3.5$, respectively (out of the total $\sim 94$ ACRN above). The other three proteins $\gamma$-Ig, Tf, and BSA show similar results, with the (ACRN-Arg, ACRN-Lys) having values of $(0.0,0.2)$, $(1.4,3.2),(0.1,2.4)$, respectively. These findings are consistent with a previous report by Kagan and coauthors, where they found that the interactions between human 
myeloperoxidase (hMPO) and the carboxylated nanotubes involved both arginine (п-пcation interaction) and tyrosine ( $\Pi-\Pi$ interaction) residues [94]. Our current simulations show that the $\Pi-\Pi$ stacking interactions are the driving forces for the competitive binding of human serum proteins onto SWCNTs.

Finally, it should be noted that carbon nanotubes often exist in much longer lengths in in vitro experiments as compared to our simulation lengths, which are typically a few to a few tens of nanometers. However, there are recent studies showing that the enzyme horseradish peroxidase (HRP) can biodegrade SWCNTs into very small fragments [95]. Therefore short SWCNTs may exist in the living body and studying SWCNTs with relatively short lengths might be of significant importance to human health as well.

\section{Conclusion}

We had investigated the effect of the CNTs on the conformation and function of proteins by using the large scale molecular dynamics simulations. WW domain, $\mathrm{SH} 3$ domains were used as examples to illustrate our ideas that the CNTs can affect the function of the proteins by either disrupting the structure of active site or shielding the active site from competing ligands. In the simulation of the SWCNT and the WW domain, it was found that the SWCNT can plug into the hydrophobic core of WW domain to form a stable protein-SWCNT complex. This insertion of the SWCNT broke the scaffold which used to bind the proline rich motifs (PRM), and thus reduced the possibility of the direct binding between the PRM and the WW domain. In the case of SH3 domain, we studied a three-way binding competition among the SWCNT, the PRM ligand, and the SH3 domain, and found that the SWCNT had a very high probability occupying the binding pocket of the ligand in the $\mathrm{SH} 3$ domain, with about $0.6 \mathrm{kcal} / \mathrm{mol}$ more favorable binding affinity than the original PRM ligand. Moreover, in most of the simulation trajectories, the ligand will be adsorbed onto the SWCNT, which may further reduce the possibility of the correct binding for the ligand onto the active site of the SH3 domain. These adverse effects of the SWCNTs on proteins, including both the disruption of the active sites and the competitive binding with the ligands, might seriously damage the original functions of proteins, suggesting the potential nanotoxicity of the SWCNT. The interactions between the SWCNTs and the hydrophobic residues, particularly the aromatic residues $(\pi-\pi$ stacking interactions), are found to play a key role in both the insertion of the SWCNT and the competitive binding with ligands. Moreover, we also simulated much larger systems, with human serum proteins interacting with SWCNTs, and found a competitive binding among all these serum proteins, with the adsorption capacity in an order of BFg $>\gamma$-Ig $>\mathrm{Tf}>\mathrm{BSA}$, in good agreement with experimental findings. Again, the $\Pi-\Pi$ stacking interactions are found to be the driving forces for the competitive binding of human serum proteins onto SWCNTs. These findings might have shed some light to the general mechanism of the interactions between hydrophobic nanoparticles and proteins.

\section{Computational method}

\section{Modeling WW domain and SWCNT}

The protein YAP65 WW domain was prepared from the Protein Data Bank (PDB code: 1JMQ, truncated to include residues 15-40), and modeled by AMBER03 force field [96]. The lengths of all those SWCNTs are $19.54 \AA$. The carbon atoms of the SWCNTs were modeled 
as uncharged Lennard-Jones particles with a cross-section of $\sigma_{\mathrm{cc}}=3.40 \AA$, and a depth of the potential well of $\varepsilon_{\mathrm{cc}}=0.36 \mathrm{~kJ} / \mathrm{mol}[56,57]$. The interactions between these carbon atoms of SWCNT and other atoms were generated by the AMBER03 force field [96]. The combined systems were then solvated in a rhombic dodecahedral periodic box with the distance between the solutes and box boundary at least $8 \AA$. The numbers of water molecules were 2694, 2709 and 2735 for the system with the SWCNT of $m=4,5$, and 6, respectively. And a $\mathrm{Cl}^{-}$was added into solution to neutralize the system.

\section{Modeling SH3 domain, Ligand and SWCNT}

The SH3 domain and the ligand were prepared from the Protein Data Bank (PDB code: 1CKB [85], residues 134-190 for the SH3 domain, and residue 1-8 for the ligand), and modeled by AMBER03 force field [96]. The carbon atoms of the SWCNT were also modeled as uncharged Lennard-Jones particles with a cross-section of $\sigma_{\mathrm{cc}}=3.40 \AA$, and a depth of the potential well of $\varepsilon_{\mathrm{cc}}=0.36 \mathrm{~kJ} / \mathrm{mol}[56,57]$. Two three-way (SWCNT + ligand + protein) binding complex systems were set up. In the first system, the initial separations of the geometric centre of the SWCNT and the ligand from that of the SH3 domain were both set as $30 \AA$, a distance long enough to avoid the starting point bias. The initial orientations of the SWCNT and the RPM ligand versus the SH3 domain were set at different directions. The resulting complex was then solvated in a rhombic dodecahedral periodic box, with the distance between the solutes and the boundary of the box at least $10 \AA$. The final complex system size is 12,378 atoms. For the second complex system, a similar procedure was followed, but with the SWCNT and ligand swapped in space (thus in the opposite orientations when compared to the first system) for effective sampling. The final system size is 13,367 atoms. The TIP3P [97] water model was used for the salvation, and three $\mathrm{Na}^{+}$were added into solution to neutralize each system.

\section{WW domain and SH3 domain MD simulations}

The MD simulations were performed by using the Gromacs package 4.0 [98]. In the simulations, the covalent bonds involving $\mathrm{H}$ atoms were constrained by the LINCS algorithm [99], allowed a time step of 2 fs. The long range electrostatic interactions were treated with the particle-mesh Ewald method (PME) with a grid spacing of $1.2 \AA$ [100]. The cutoff for the van der Waals interaction was set to $10 \AA$. After energy minimization, all the systems were equilibrated by MD simulations for 200 ps at a constant pressure of 1 bar and temperature of $298 \mathrm{~K}$ using Berendsen coupling [101]. Then the production simulations were performed in the NVT ensemble at $298 \mathrm{~K}$.

\section{Human serum proteins interacting with SWCNT}

The human blood serum proteins simulated in this work were taken from the Protein Data Bank and Swiss-Model Repository as BFg with PDB ID: 1DEQ, $\gamma$-Ig with PDB ID: 3HR5, Tf with PDB ID: 2HAV, and BSA with SWISS-MODEL ID: 432779d395a52bfc9f6574bc3e98afcd_1. All SWCNTs used in the molecular dynamics (MD) simulations for the adsorption of CNT for proteins $\mathrm{BFg}$, Ig, $\mathrm{Tf}$, and BSA were armchair CNT $(14,14)$ with diameter as around $2.0 \mathrm{~nm}$, which were consistent with the experimental ones. The geometrical coordinate parameters of CNT were generated by using Nanotube Modeler software (www.jcrystal.com/products/wincnt/).

MD simulations were performed by NAMD [102] of version 2.6. The various blood proteins were modeled by CHARMM 32b1 force field [103]. Carbon atoms of SWCNTs were assumed to be type CA with Lennard-Jones parameters $\varepsilon_{c c}=-0.0700 \mathrm{kcal} / \mathrm{mol}$, 
$R_{\min } / 2=1.9924 \AA$. The interaction between these carbon atoms of SWCNTs and other atoms were generated by CHARMM $32 \mathrm{~b} 1$ force field. The various blood proteins were wellseparated from SWCNT with the minimum distances up to $40.0 \AA$ initially. All systems were solvated in periodic TIP3P modeled water box, and neutralized by adding sodium and chloride ions with $0.2 \mathrm{M}$ salt concentration as physiological condition, with system sizes up to 1,058,598 atoms (see Fig. 12 for one example of BFg).

Simulations were performed at constant temperature of $310 \mathrm{~K}$, and pressure of $1 \mathrm{~atm}$, with the PME method for long-range electrostatic interactions and time step of $2 \mathrm{fs}$. The cutoff for the Van der Waals interaction was set to $12 \AA$. All systems were simulated in the NPT ensemble for more than 150 ns. Visual Molecular Dynamics (VMD 1.8.7.) graphics viewer software was utilized to illustrate the adsorption of proteins and SWCNT in different representations.

\section{Acknowledgement}

We thank Dr. Peng Xiu, Dr. Chunlei Wang, Dr. Payel Das, Dr. Seung-gu Kang, and Prof. Bruce Berne for helpful discussions. This research is supported in part by grants from NNSFC (10825520), NBRPC (973 Program: 2007CB936000 and 2007CB814800), Shanghai Leading Academic Discipline Project (B111), and Shanghai Supercomputer Center of China. RZ acknowledges the support from the IBM BlueGene Science Program.

\section{References}

[1] Iijima, S., Helical microtubules of graphitic carbon. Nature, 1991. 354: p. 56-58.

[2] Li, W.Z., et al., Large-scale synthesis of aligned carbon nanotubes. Science, 1996. 274: p. 1701-1703.

[3] Prato, M., K. Kostarelos, and A. Bianco, Functionalized carbon nanotubes in drug design and discovery. Accounts of Chemical Research, 2008. 41(1): p. 60-68.

[4] Bhirde, A.A., et al., Targeted killing of cancer cells in vivo and in vitro with EGF-directed carbon nanotube-based drug delivery. ACS Nano, 2009. 3(2): p. 307-316.

[5] Thakare, V.S., et al., Carbon nanotubes in cancer theragnosis. Nanomedicine, 2010. 5(8): p. 1277-1301.

[6] Zanello, L.P., et al., Bone cell proliferation on carbon nanotubes. Nano Letters, 2006. 6(3): p. 562567.

[7] Tu, X., et al., DNA sequence motifs for structure-specific recognition and separation of carbon nanotubes. Nature, 2009. 460(7252): p. 250-253.

[8] Liu, N., et al., Carbon Nanotubes for Electrochemical and Electronic Biosensing Applications. NanoScience in Biomedicine, 2009: p. 205-246.

[9] Thayer, A.M., Carbon nanotubes by the metric ton. Chemical \& Engineering News, 2007. 85(46): p. 29-35.

[10] Service, R.F., Is nanotechnology dangerous? Science, 2000. 290: p. 1526-1527.

[11] Gilbert, N., Nanoparticle safety in doubt. Nature, 2009. 460: p. 937-937.

[12] Nel, A., et al., Toxic potential of materials at the nanolevel. Science, 2006. 311: p. 622-627.

[13] Zhao, Y., G. Xing, and Z. Chai, Nanotoxicology: Are carbon nanotubes safe? Nature Nanotechnology, 2008. 3(4): p. 191-192.

[14] Maynard, A.D., et al., Safe handling of nanotechnology. Nature, 2006. 444(7117): p. 267-269.

[15] Bi, S.P., J. Zhang, and J.J. Cheng, Call from China for joint nanotech toxicity-testing effort. Nature, 2009. 461(7264): p. 593-593. 
[16] Donaldson, K. and C.A. Poland, Nanotexicology: New insights into nanotubes. Nature Nanotechnology, 2009. 4(11): p. 708-710.

[17] Buzea, C., I.I. Pacheco, and K. Robbie, Nanomaterials and nanoparticles: Sources and toxicity. Biointerphases, 2007. 2(4): p. MR17-MR71.

[18] Maynard, A.D., et al., Exposure to carbon nanotube material: Aerosol release during the handling of unrefined single-walled carbon nanotube material. Journal of Toxicology and Environmental Health-Part A, 2004. 67: p. 87-107.

[19] Ryman-Rasmussen, J.P., et al., Inhaled carbon nanotubes reach the subpleural tissue in mice. Nature Nanotechnology, 2009. 4: p. 747-751.

[20] Kolosnjaj, J., H. Szwarc, and F. Moussa, Toxicity studies of carbon nanotubes. Advances in Experimental Medicine and Biology, 2007. 620: p. 181-204.

[21] Porter, A.E., et al., Direct imaging of single-walled carbon nanotubes in cells. Nature Nanotechnology, 2007. 2: p. 713-717.

[22] Ma-Hock, L., et al., Inhalation toxicity of multiwall carbon nanotubes in rats exposed for 3 months. Toxicological Sciences, 2009. 112: p. 468-481.

[23] Schipper, M.L., et al., A pilot toxicology study of single-walled carbon nanotubes in a small sample of mice. Nature Nanotechnology, 2008. 3(4): p. 216-221.

[24] Shvedova, A.A., et al., Unusual inflammatory and fibrogenic pulmonary responses to singlewalled carbon nanotubes in mice. American Journal of Physiology - Lung Cellular and Molecular Physiology, 2005. 289: p. L698-708.

[25] Mitchell, L.A., et al., Mechanisms for how inhaled multiwalled carbon nanotubes suppress systemic immune function in mice. Nature Nanotechnology, 2009. 4: p. 451-456.

[26] Li, Z., et al., Cardiovascular effects of pulmonary exposure to single-wall carbon nanotubes. Environmental Health Perspectives, 2007. 115: p. 377-382.

[27] Poland, C.A., et al., Carbon nanotubes introduced into the abdominal cavity of mice show asbestos-like pathogenicity in a pilot study. Nature Nanotechnology, 2008. 3: p. 423-428.

[28] Bai, Y.H., et al., Repeated administrations of carbon nanotubes in male mice cause reversible testis damage without affecting fertility. Nature Nanotechnology, 2010. 5(9): p. 683-689.

[29] Balavoine, F., et al., Helical crystallization of proteins on carbon nanotubes: A first step towards the development of new biosensors. Angewandte Chemie International Edition, 1999. 38(13-14): p. 1912-1915.

[30] Park, K.H., et al., Single-walled carbon nanotubes are a new class of ion channel blockers. Journal of Biological Chemistry, 2003. 278: p. 50212-50216.

[31] Karajanagi, S.S., et al., Structure and function of enzymes adsorbed onto single-walled carbon nanotubes. Langmuir, 2004. 20(26): p. 11594-11599.

[32] Goldberg-Oppenheimer, P. and O. Regev, Exploring a nanotube dispersion mechanism with gold-labeled proteins via Cryo-TEM imaging. Small, 2007. 3(11): p. 1894-1899.

[33] Wang, S., et al., Peptides with selective affinity for carbon nanotubes. Nature Materials, 2003. 2: p. 196-200.

[34] Zheng, L.F., D. Jain, and P. Burke, Nanotube-peptide interactions on a silicon chip. Journal of Physical Chemistry C, 2009. 113: p. 3978-3985.

[35] Cedervall, T., et al., Understanding the nanoparticle-protein corona using methods to quantify exchange rates and affinities of proteins for nanoparticles. Proceedings of the National Academy of Sciences of the United States of America, 2007. 104: p. 2050-2055.

[36] Klein, J., Probing the interactions of proteins and nanoparticles. Proceedings of the National Academy of Sciences of the United States of America, 2007. 104: p. 2029-2030.

[37] Rocker, C., et al., A quantitative fluorescence study of protein monolayer formation on colloidal nanoparticles. Nature Nanotechnology, 2009. 4: p. 577-580. 
[38] Duan, Y. and P.A. Kollman, Pathways to a protein folding intermediate observed in a 1microsecond simulation in aqueous solution. Science, 1998. 282: p. 740-744.

[39] Levy, Y., P.G. Wolynes, and J.N. Onuchic, Protein topology determines binding mechanism. Proceedings of the National Academy of Sciences of the United States of America, 2004. 101: p. 511-516.

[40] Snow, C.D., et al., Absolute comparison of simulated and experimental protein-folding dynamics. Nature, 2002. 420(6911): p. 102-106.

[41] Zhou, R.H., et al., Hydrophobic collapse in multidomain protein folding. Science, 2004. 305(5690): p. 1605-1609.

[42] Liu, P., et al., Observation of a dewetting transition in the collapse of the melittin tetramer. Nature, 2005. 437(7055): p. 159-162.

[43] Zuo, G., J. Hu, and H. Fang, Effect of the ordered water on protein folding: an off-lattice Gō-like model study. Physical Review E (Statistical, Nonlinear, and Soft Matter Physics), 2009. 79(3 Pt 1): p. 031925.

[44] Mayor, U., et al., The complete folding pathway of a protein from nanoseconds to microseconds. Nature, 2003. 421: p. 863-867.

[45] Mirny, L. and E. Shakhnovich, Protein folding theory: From lattice to all-atom models. Annual Review of Biophysics and Biomolecular Structure, 2001. 30: p. 361-396.

[46] Garcia, A.E. and D. Paschek, Simulation of the pressure and temperature folding/unfolding equilibrium of a small RNA hairpin. Journal of the American Chemical Society, 2008. 130: p. 815-816.

[47] Miyashita, N., J.E. Straub, and D. Thirumalai, Structures of beta-Amyloid Peptide 1-40, 1-42, and 1-55-the 672-726 Fragment of APP-in a Membrane Environment with Implications for Interactions with gamma-Secretase. Journal of the American Chemical Society, 2009. 131: p. 17843-17852.

[48] Hua, L., et al., Dynamics of water confined in the interdomain region of a multidomain protein. Journal of Physical Chemistry B, 2006. 110: p. 3704-3711.

[49] Liu, P., et al., Hydrophobic aided replica exchange: an efficient algorithm for protein folding in explicit solvent. Journal of Physical Chemistry B, 2006. 110: p. 19018-19022.

[50] Zhou, R., Exploring the protein folding free energy landscape: coupling replica exchange method with P3ME/RESPA algorithm. Journal of Molecular Graphics \& Modelling 2004. 22: p. 451-463.

[51] Zhou, R.H., et al., Destruction of long-range interactions by a single mutation in lysozyme. Proceedings of the National Academy of Sciences of the United States of America, 2007. 104(14): p. 5824-5829.

[52] Gao, Y.Q., W. Yang, and M. Karplus, A structure-based model for the synthesis and hydrolysis of ATP by F1-ATPase. Cell, 2005. 123: p. 195-205.

[53] Karplus, M., et al., Protein structural transitions and their functional role. Philos Transact A Math Phys Eng Sci, 2005. 363: p. 331-355; discussion 355-356.

[54] Roitberg, A.E., A. Okur, and C. Simmerling, Coupling of replica exchange simulations to a nonBoltzmann structure reservoir. Journal of Physical Chemistry B, 2007. 111: p. 2415-2418.

[55] Li, W.F., et al., Metal-coupled folding of cys2his2 zinc-finger. Journal of the American Chemical Society, 2008. 130(3): p. 892-900.

[56] Gong, X.J., et al., A charge-driven molecular water pump. Nature Nanotechnology, 2007. 2: p. 709-712.

[57] Hummer, G., J.C. Rasaiah, and J.P. Noworyta, Water conduction through the hydrophobic channel of a carbon nanotube. Nature, 2001. 414: p. 188-190.

[58] Tu, Y.S., et al., Water-mediated signal multiplication with Y-shaped carbon nanotubes. Proceedings of the National Academy of Sciences of the United States of America, 2009. 106: p. 18120-18124. 
[59] Li, J.Y., et al., Electrostatic gating of a nanometer water channel. Proceedings of the National Academy of Sciences of the United States of America, 2007. 104: p. 3687-3692.

[60] Giovambattista, N., et al., Hydrophobicity of protein surfaces: Separating geometry from chemistry. Proceedings of the National Academy of Sciences of the United States of America, 2008. 105: p. 2274-2279.

[61] Shen, J.-W., et al., Induced stepwise conformational change of human serum albumin on carbon nanotube surfaces. Biomaterials, 2008. 29(28): p. 3847-3855.

[62] Zuo, G.H., et al., Plugging into proteins: Poisoning protein function by a hydrophobic nanoparticle. ACS Nano, 2010. 4(12): p. 7508-7514.

[63] Chen, H.I. and M. Sudol, The WW domain of Yes-associated protein binds a proline-rich ligand that differs from the consensus established for Src homology 3-binding modules. Proceedings of the National Academy of Sciences of the United States of America, 1995. 92(17): p. 7819-7823.

[64] Garrus, J.E., et al., Tsg101 and the vacuolar protein sorting pathway are essential for HIV-1 budding. Cell, 2001. 107: p. 55-65.

[65] Lu, P.J., et al., The prolyl isomerase Pin1 restores the function of Alzheimer-associated phosphorylated tau protein. Nature, 1999. 399(6738): p. 784-788.

[66] Sudol, M., et al., Characterization of the mammalian YAP (yes-associated protein) gene and Its role in defining a novel protein module, the WW domain. Journal of Biological Chemistry, 1995. 270(24): p. 14733-14741.

[67] Zheng, H.W., et al., The prolyl isomerase Pin1 is a regulator of p53 in genotoxic response. Nature, 2002. 419(6909): p. 849-853.

[68] Macias, M.J., et al., Structural analysis of WW domains and design of a WW prototype. Nature Structural Molecular Biology, 2000. 7(5): p. 375-379.

[69] Huang, X., et al., Structure of a WW domain containing fragment of dystrophin in complex with beta-dystroglycan. Nature Structural Biology, 2000. 7(8): p. 634-638.

[70] Sudol, M., Structure and function of the WW domain. Progress in Biophysics and Molecular Biology, 1996. 65(1-2): p. 113-132.

[71] Macias, M.J., et al., Structure of the WW domain of a kinase-associated protein complexed with a proline-rich peptide. Nature, 1996. 382: p. 646-649.

[72] Dill, K., Dominant forces in protein folding. Biochemistry, 1990. 29: p. 7133-7155.

[73] Nadassy, K., S.J. Wodak, and J. Janin, Structural features of protein-nucleic acid recognition sites. Biochemistry, 1999. 38: p. 1999-2017.

[74] Kabsch, W. and C. Sander, Dictionary of protein secondary structure: pattern recognition of hydrogen-bonded and geometrical features. Biopolymers, 1983. 22: p. 2577-2637.

[75] Braden, B.C., et al., X-ray crystal structure of an anti-Buckminsterfullerene antibody fab fragment: biomolecular recognition of $C(60)$. Proceedings of the National Academy of Sciences of the United States of America, 2000. 97: p. 12193-12197.

[76] Noon, W.H., Y. Kong, and J. Ma, Molecular dynamics analysis of a buckyball-antibody complex. Proceedings of the National Academy of Sciences of the United States of America, 2002. 99 Suppl 2: p. 6466-6470.

[77] Venter, J.C., et al., The sequence of the human genome. Science, 2001. 291(5507): p. 1304-1351.

[78] Ball, L.J., et al., Recognition of proline-rich motifs by protein-protein-interaction domains. Angewandte Chemie International Edition, 2005. 44(19): p. 2852-2869.

[79] Macias, M.J., S. Wiesner, and M. Sudol, WW and SH3 domains, two different scaffolds to recognize proline-rich ligands. FEBS Letters, 2002. 513(1): p. 30-37.

[80] Bucciantini, M., et al., Inherent toxicity of aggregates implies a common mechanism for protein misfolding diseases. Nature, 2002. 416(6880): p. 507-511.

[81] Gorina, S. and N.P. Pavletich, Structure of the p53 tumor suppressor bound to the ankyrin and SH3 domains of 53BP2. Science, 1996. 274(5289): p. 1001-1005. 
[82] Zarrinpar, A., R.P. Bhattacharyya, and W.A. Lim, The structure and function of proline recognition domains. Sci STKE, 2003. 2003(179): p. RE8.

[83] Li, X.F., et al., Structural basis of robo proline-rich motif recognition by the srGAP1 Src homology 3 domain in the slit-robo signaling pathway. Journal of Biological Chemistry, 2006. 281(38): p. 28430-28437.

[84] Kobashigawa, Y., et al., Structural basis for the transforming activity of human cancer-related signaling adaptor protein CRK. Nature Structural \& Molecular Biology, 2007. 14(6): p. 503-510.

[85] Wu, X.D., et al., Structural basis for the specific interaction of lysine-containing proline-rich peptides with the N-terminal SH3 domain of c-Crk. Structure, 1995. 3: p. 215-226.

[86] Nguyen, J.T., et al., Exploiting the basis of proline recognition by SH3 and WW domains: Design of n-substituted inhibitors. Science, 1998. 282: p. 2088-2092.

[87] Ahmad, M., W. Gu, and V. Helms, Mechanism of fast peptide recognition by SH3 domains13. Angewandte Chemie International Edition, 2008. 47(40): p. 7626-7630.

[88] Freedman, D., R. Pisani, and R. Purves, Statistics. 2007: W.W. Norton \& Co.

[89] Brown, S., T.S. Jespersen, and J. Nygard, A genetic analysis of carbon-nanotube-binding proteins. Small, 2008. 4(4): p. 416-420.

[90] Tomasio, S.D. and T.R. Walsh, Atomistic modelling of the interaction between peptides and carbon nanotubes. Molecular Physics, 2007. 105(2-3): p. 221-229.

[91] Selzer, T., S. Albeck, and G. Schreiber, Rational design of faster associating and tighter binding protein complexes. Nature Structural Biology, 2000. 7(7): p. 537-541.

[92] Schreiber, G. and A.R. Fersht, Rapid, electrostatically assisted association of proteins. Nature Structural Biology, 1996. 3(5): p. 427-431.

[93] Ge, C., et al., $\pi-\pi$ stacking interaction driven competitive adsorption of human blood proteins on single-wall carbon nanotubes and its implications for resulting cytotoxicity. Nature Nanotechnology, 2011: p. submitted.

[94] Kagan, V.E., et al., Carbon nanotubes degraded by neutrophil myeloperoxidase induce less pulmonary inflammation. Nature Nanotechnology, 2010. 5(5): p. 354-359.

[95] Allen, B.L., et al., Biodegradation of single-walled carbon nanotubes through enzymatic catalysis. Nano Letters, 2008. 8: p. 3899-2903.

[96] Duan, Y., et al., A point-charge force field for molecular mechanics simulations of proteins based on condensed-phase quantum mechanical calculations. Journal of Computational Chemistry, 2003. 24: p. 1999-2012.

[97] Jorgensen, W.L., et al., Comparison of Simple Potential Functions for Simulating Liquid Water. Journal of Chemical Physics, 1983. 79(2): p. 926-935.

[98] Hess, B., et al., GROMACS 4: Algorithms for highly efficient, load-balanced, and scalable molecular simulation. Journal of Chemical Theory and Computation, 2008. 4: p. 435-447.

[99] Hess, B., P-LINCS: A parallel linear constraint solver for molecular simulation. Journal of Chemical Theory and Computation, 2008. 4(1): p. 116-122.

[100] Darden, T., D. York, and L. Petersen, Particle mesh Ewald: an $N \log (N)$ method for Ewald sums in large systems. The Journal of Chemical Physics, 1993. 98: p. 10089-10092.

[101] Berendsen, H.J.C., et al., Molecular dynamics with coupling to an external bath. The Journal of Chemical Physcis, 1984. 81(8): p. 3684-3690.

[102] Phillips, J.C., et al., Scalable molecular dynamics with NAMD. Journal of Computational Chemistry, 2005. 26(16): p. 1781-1802.

[103] Feller, S.E. and A.D. MacKerell Jr, An improved empirical potential energy function for molecular simulations of phospholipids. Journal of Physical Chemistry B, 2000. 104(31): p. 7510-7515. 


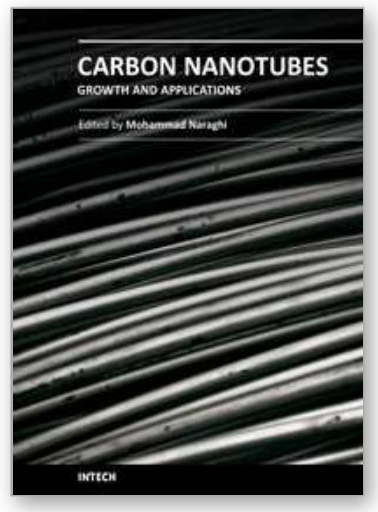

\section{Carbon Nanotubes - Growth and Applications}

Edited by Dr. Mohammad Naraghi

ISBN 978-953-307-566-2

Hard cover, 604 pages

Publisher InTech

Published online 09, August, 2011

Published in print edition August, 2011

Carbon Nanotubes are among the strongest, toughest, and most stiff materials found on earth. Moreover, they have remarkable electrical and thermal properties, which make them suitable for many applications including nanocomposites, electronics, and chemical detection devices. This book is the effort of many scientists and researchers all over the world to bring an anthology of recent developments in the field of nanotechnology and more specifically CNTs. In this book you will find:

- Recent developments in the growth of CNTs

- Methods to modify the surfaces of CNTs and decorate their surfaces for specific applications

- Applications of CNTs in biocomposites such as in orthopedic bone cement

- Application of CNTs as chemical sensors

- CNTs for fuelcells

- Health related issues when using CNTs

\section{How to reference}

In order to correctly reference this scholarly work, feel free to copy and paste the following:

Guanghong Zuo, Haiping Fang and Ruhong Zhou (2011). Nanotoxicity: Exploring the Interactions Between Carbon Nanotubes and Proteins, Carbon Nanotubes - Growth and Applications, Dr. Mohammad Naraghi (Ed.), ISBN: 978-953-307-566-2, InTech, Available from: http://www.intechopen.com/books/carbon-nanotubesgrowth-and-applications/nanotoxicity-exploring-the-interactions-between-carbon-nanotubes-and-proteins

\section{INTECH}

open science | open minds

\section{InTech Europe}

University Campus STeP Ri

Slavka Krautzeka 83/A

51000 Rijeka, Croatia

Phone: +385 (51) 770447

Fax: +385 (51) 686166

www.intechopen.com

\section{InTech China}

Unit 405, Office Block, Hotel Equatorial Shanghai

No.65, Yan An Road (West), Shanghai, 200040, China

中国上海市延安西路65号上海国际贵都大饭店办公楼405单元

Phone: +86-21-62489820

Fax: $+86-21-62489821$ 
(C) 2011 The Author(s). Licensee IntechOpen. This chapter is distributed under the terms of the Creative Commons Attribution-NonCommercialShareAlike-3.0 License, which permits use, distribution and reproduction for non-commercial purposes, provided the original is properly cited and derivative works building on this content are distributed under the same license. 